\title{
Turbulent viscosity in clumpy accretion disks
}

\section{Supernova driven turbulence in the Galaxy}

\author{
B. Vollmer and T. Beckert
}

\begin{abstract}
Max-Planck-Institut für Radioastronomie, Auf dem Hügel 69, 53121 Bonn, Germany
\end{abstract}
Received 17 July 2002 / Accepted 18 March 2003

\begin{abstract}
An analytical model for a turbulent clumpy gas disk is presented where turbulence is maintained by the energy input due to supernovae. Expressions for the disk parameters, global filling factors, molecular fractions, and star formation rates are given as functions of the Toomre parameter $Q$, the ratio between the cloud size and the turbulent driving length scale $\delta$, the mass accretion rate within the disk $\dot{M}$, the constant of molecule formation $\alpha$, the disk radius, the angular velocity, and its radial derivative. Two different cases are investigated: a dominating stellar disk and a self-gravitating gas disk in $z$ direction. The turbulent driving wavelength is determined in a first approach by energy flux conservation, i.e. the supernovae energy input is transported by turbulence to smaller scales where it is dissipated. The results are compared to those of a fully gravitational model. For $Q=1$ and $\delta=1$ both models are consistent with each other. In a second approach the driving length scale is directly determined by the size of supernovae remnants. Both models are applied to the Galaxy and can reproduce its integrated and local gas properties. The influence of thermal and magnetic pressure on the disk structure is investigated. We infer $Q \sim 1$ and $\dot{M} \sim 0.05-0.1 M_{\odot} \mathrm{yr}^{-1}$ for the Galaxy.
\end{abstract}

Key words. ISM: clouds - ISM: structure - Galaxy: structure - galaxies: ISM

\section{Introduction}

The interstellar medium (ISM) is of turbulent nature. Its structure is usually described as hierarchical (Scalo 1985) over length scales of several magnitudes up to $\sim 100 \mathrm{pc}$. The neutral phase of the ISM is not uniform but of fractal nature (Elmegreen \& Falgarone 1996).

In a widely accepted picture (see, e.g. Kulkarni \& Heiles 1988; Spitzer 1990; McKee 1995), the ISM consists of 5 different phases that are listed in Table 1 . About $80 \%$ of the total gas mass is neutral and $50 \%$ is in form of clouds or filaments. The scale height of the cold material is about 4 times smaller than that of the warm gas. The hot medium is heated and ionized by direct thermal energy input due to supernova (SN) explosions. The warm medium is heated and ionized by SN remnants, stellar winds and radiation. The main energy sources driving interstellar turbulence and thus causing the multiphase structure of the ISM are (i) SN explosions (see, e.g., Ruzmaikin et al. 1988) or (ii) the galaxy's gravitational potential and local gravitational instabilities (Wada et al. 2002).

Several attempts have been made to include SN explosions into hydrodynamical simulations:

Rosen \& Bregman (1995) computed two-dimensional simulations with two cospatial fluids representing stars and gas. Star formation, stellar mass loss, stellar winds and SN were included. These simulations created a three-phase medium with

Send offprint requests to: $\mathrm{B}$. Vollmer,

e-mail: bvollmer@mpifr-bonn.mpg.de filaments of dense, cold and warm gas surrounding bubbles of hot gas.

Korpi et al. (1999) simulated the local ISM heated and stirred by $\mathrm{SNe}$ using a three-dimensional, non-ideal MHD model in a box of $\sim 1 \mathrm{kpc}$. They used a prescription for the $\mathrm{SN}$ rate deduced from observations. These simulations showed a stationary multicomponent structure of the gas in a state of developed turbulence. They found a turbulent cell size of $60 \mathrm{pc}$ in the warm phase.

Gazol-Patino \& Passot (1999) studied the role of superbubbles in the evolution of the ISM at the kiloparsec scale. The model incorporated the fully compressible MHD equations, including parameterized cooling and heating, the Coriolis force, shear, self-gravitation, and energy input by $\mathrm{SNe}$ in 2.5 dimensions. The SN rate was determined by the gas flow itself. Their simulations also lead to a state of highly compressible turbulence. The inclusion of $\mathrm{SNe}$ into the model gave rise to superbubbles whose presence leads to a more intermittent cycle of star formation.

Wada \& Norman (2001) made high-resolution twodimensional hydrodynamic simulations of the ISM including star formation and energy input by SNe. In their simulations a globally stable multiphase ISM formed. They found an energy spectrum of $E(k) \propto k^{-2}$ and a turbulent length scale of $20-100 \mathrm{pc}$.

The influence of SN explosions on the ISM was studied by de Avillez \& Mac Low (2001) with a three-dimensional hydrodynamical code using adaptive mesh refinement. 
Table 1. The properties of the different phases of the ISM. $T$ : temperature, $n$ : density, $\langle n\rangle$ : mean density, $v$ : dispersion velocity, $\Phi_{\mathrm{V}}$ : volume filling factor, $H$ : scale height, $M$ : percentage of the total gas mass (from Boulares \& Cox 1990).

\begin{tabular}{lccccccc}
\hline \hline & $T(\mathrm{~K})$ & $n\left(\mathrm{~cm}^{-3}\right)$ & $\left\langle n>\left(\mathrm{cm}^{-3}\right)\right.$ & $v_{\mathrm{rms}}\left(\mathrm{km} \mathrm{s}^{-1}\right)$ & $\Phi_{\mathrm{V}}$ & $H(\mathrm{pc})$ & $M / M_{\mathrm{tot}}(\%)$ \\
\hline Hot ionized & $\sim 10^{6}$ & $\ldots$ & 0.002 & $\ldots$ & 0.5 & 3000 & 4 \\
Warm ionized & $\sim 8000$ & $0.3-10$ & 0.025 & $\sim 10$ & 0.2 & 900 & 14 \\
Warm neutral & $\sim 8000$ & $0.1-10$ & 0.1 & $\sim 10$ & 0.3 & 400 & 31 \\
Cold neutral & $\sim 100$ & $10-1000$ & 0.3 & $\sim 6$ & 0.02 & 140 & 25 \\
Molecular & $\sim 10$ & $>100$ & 0.6 & $\sim 6$ & 0.001 & 70 & 26 \\
\hline
\end{tabular}

SN explosions were set up at a rate comparable to observations. They showed that hot gas that is not evacuated through chimneys expands into the cooler gas of the thick disk building mushroom-shaped structures.

Even though it is clear that SN explosions represent the dominant energy input in galaxies with a normal star formation rate, all these simulations show how SNe can effectively drive ISM turbulence in detail.

In order to form massive stars which have a SN explosion at the end of their lifetime, self-gravitation is the starting point. Self-gravitation drives star formation and subsequently SN explosions.

In a previous paper (Vollmer \& Beckert 2002) we have elaborated a model of an equilibrium disk where turbulence is generated by instabilities involving self-gravitation and maintained by the energy input from differential rotation and mass transfer. The model only involves gravity. Its application to the Galaxy showed good agreement with observations.

In this article we extend our previous model by including $\mathrm{SNe}$ as an additional energy input to drive ISM turbulence. We give the basic picture in Sect. 2. The equations are presented in Sect. 3. Two different models with different vertical pressure equilibria are analyzed: (i) a model of a selfgravitating gas disk in $z$ direction (SGZ model) and (ii) a model with a dominating stellar disk mass (DSD model). We introduce an energy flux conservation equation that relates the energy flux transported by turbulence to the energy input due to SN explosions. In Sect. 4 we give analytical expressions for the disk properties and show results for characteristic values of the input parameters. The SGZ model is compared in Sect. 5 to the fully gravitational model of Vollmer \& Beckert (2002). In Sect. 6 the energy flux conservation is replaced by an equation relating the turbulent driving length scale directly to the size of a SN remnant (SNR model). We apply the SGZ and SNR model to the Galaxy in Sect. 7. In Sect. 8 a realistic gravitational potential for the galaxy is introduced and the inclusion of magnetic and thermal pressure into the models is discussed. Section 10 investigates disks with $Q>1$ and $Q<1$. The summary and conclusions are given in Sect. 11.

\section{The basic picture}

We consider the warm, cold, and molecular phases of the ISM as one gas that can undergo phase changes according to internal and external conditions. Internal conditions are pressure, metalicity, molecule formation, and turbulent time scales; external conditions are stellar radiation field (UV, X-rays, cosmic rays), stellar winds, and SN explosions with subsequent shock formation. In this approach we neglect the thermal balance due to radiative heating and cooling of the ISM.

The ISM is assumed to be turbulent. Turbulence is driven by $\mathrm{SNe}$ energy input at a characteristic length scale $l_{\text {driv }}$. This length scale might be identified as the characteristic length scale of a SN bubble or alternatively that of the interaction of $\mathrm{SN}$ bubbles. The disk scale height is determined unambiguously by the turbulent pressure $p_{\text {turb }}=\rho v_{\text {turb }}^{2}$, where $\rho$ is the average density and $v_{\text {turb }}$ the turbulent velocity in the disk. We thus neglect thermal, cosmic ray, and magnetic pressure. The interaction of $\mathrm{SN}$ bubbles leads to viscous transport of angular momentum. We assume that the energy input due to $\mathrm{SNe}$ is dissipated by viscous heating.

Thus, we consider a gaseous turbulent accretion disk in a given gravitational potential $\Phi$ which gives rise to an angular velocity $\Omega=\sqrt{R^{-1} \frac{\mathrm{d} \Phi}{\mathrm{d} R}}$. The Toomre parameter (Toomre 1964) is treated as a constant but free parameter for the whole disk

$Q=\frac{v_{\text {turb }} \kappa}{\pi G \Sigma}$,

with the restriction $Q \geq 1$, where $G$ is the gravitational constant, $\Sigma$ the average gas surface density, and $\kappa$ the local epicyclic frequency.

\section{The equations}

\subsection{The volume filling factor}

We compare the crossing time of a turbulent cloud to the gravitational free fall time in order to derive an expression for the volume filling factor $\phi_{\mathrm{V}}$. The characteristic turbulent time scale of clouds whose size is a factor $\delta^{-\frac{3}{1+D}}$ smaller than the driving length scale $l_{\mathrm{cl}}=\delta^{-\frac{3}{1+D}} l_{\text {driv }}$ is

$t_{1}=\delta^{-\frac{2}{3}-\frac{3-D}{3}} l_{\text {driv }} / v_{\text {turb }}$,

where $D$ is the fractal dimension (see, e.g., Frisch 1995). The local gravitational free fall time is given by

$$
t_{\mathrm{ff}}=\sqrt{\frac{3 \pi}{32 G \rho_{\mathrm{cl}}}},
$$

where $\rho_{\mathrm{cl}}$ is the density of a single cloud, which is related to the overall disk density $\rho$ by the volume filling factor $\phi_{\mathrm{V}}$ : $\rho_{\mathrm{cl}}=\phi_{\mathrm{V}}^{-1} \rho$. The clouds become self-gravitating for $t_{1}=t_{\mathrm{ff}}^{1}$. We assume $D=2$ for a compressible, selfgravitating fluid, which is close to the findings of Elmegreen \& Falgarone (1996). 


\subsection{The viscosity prescription}

In general the viscosity $v$ is defined as the product of the characteristic velocity $v$ and the characteristic length scale $l$ of the system: $v=v l$. We assume that turbulence becomes intermittent due to self-gravitation of cold gas clouds. There are two simple frameworks for understanding intermittency: (i) based on velocity (ii) based on dissipation.

Within the framework of intermittency based on dissipation (Frisch 1995), the local spatial average of the energy dissipation rate per mass unit $\epsilon$, which is constant over all length scales in the case of a Kolmogorov-like turbulence, depends explicitly on the length scale

$\frac{\epsilon_{l}}{v_{\text {turb }}^{3} / l_{\text {driv }}} \sim\left(\frac{l}{l_{\text {driv }}}\right)^{3-D}$

where $D$ is the fractal dimension ( $D=3$ for a Kolmogorovlike turbulence), $l_{\text {driv }}$ is the driving length scale. This can be achieved if there is an energy sink in the turbulent cascade. We identify the self-gravitation of cold gas clouds as this energy sink, i.e. during the contraction of a cloud kinematic energy is transformed into heat and radiated away so that the cloud can further shrink. Thus self-gravity gives rise to intermittency in the ISM turbulence.

Within the framework of intermittency based on velocity, intermittency can be understood as a decreasing volume filling factor of turbulent eddies with decreasing length scale. This can also be achieved by self-gravitation, because self-gravitating clouds are smaller than diffuse clouds of the same mass.

In the case of intermittent turbulence the turbulent viscosity $v=v_{\text {turb }} l_{\text {driv }}$ overestimates the true viscosity and has to be decreased by a factor $\gamma$ :

$v=\gamma v_{\text {turb }} l_{\text {driv }}$.

This factor is related to self-gravitation, i.e. the free fall time of the disk in $z$ direction. Based on these arguments we make the hypothesis that the characteristic velocity is determined by the disk height $H$ and the averaged local free fall time $t_{\mathrm{ff}}^{\mathrm{H}}$ : $v=H / t_{\mathrm{ff}}^{\mathrm{H}}$. The appropriate length scale is the turbulent driving length scale $l_{\text {driv }}$.

Thus we obtain:

$v=\frac{H}{t_{\mathrm{ff}}^{\mathrm{H}}} l_{\text {driv }}$.

The free fall time is given by $t_{\mathrm{ff}}^{\mathrm{H}}=\sqrt{(3 \pi) /(32 G \rho)}$, where $\rho$ is the averaged midplane density of the disk. Using the local free fall time (Eq. (3)) with $\rho_{\mathrm{cl}}=\phi_{\mathrm{V}}^{-1} \rho$, where $\phi_{\mathrm{V}}$ is the volume filling factor of self-gravitating clouds (Sect. 3.1). The characteristic velocity can then be written as

$v=\frac{H}{t_{\mathrm{ff}}^{\mathrm{H}}}=\frac{H}{l_{\text {driv }}} \sqrt{\phi_{\mathrm{V}}} \delta v_{\text {turb }}$.

Inserting this velocity into Eq. (6) gives

$v=H \sqrt{\phi_{\mathrm{V}}} \delta v_{\text {turb }}$.

Thus, the factor $\gamma$ that is due to intermittency is

$\gamma=\frac{H}{l_{\text {driv }}} \sqrt{\phi_{\mathrm{V}}} \delta$.

We will call it the viscosity intermittence factor.

\subsection{Angular momentum equation}

The viscosity prescription given above is essential for mass accretion and the transport of angular momentum. In a steady state accretion disk, the mass accretion rate is

$\dot{M}=2 \pi R \Sigma\left(-v_{\text {rad }}\right)$,

where $v_{\text {rad }}$ is the radial velocity. The angular momentum equation can be integrated giving

$v \Sigma=-\frac{\dot{M}}{2 \pi R} \Omega\left(\frac{\partial \Omega}{\partial R}\right)^{-1}$.

Furthermore, we use

$\Sigma=\rho H$

for the surface density of the disk, where $\rho$ is the average mass density at the midplane.

\subsection{The vertical pressure equilibrium}

We assume that the only pressure which counterbalances gravitation in the vertical direction is the turbulent pressure $p_{\text {turb }}=$ $\rho v_{\text {turb }}^{2}$. We distinguish two cases for the gravitational force density in the vertical $z$ direction:

1. $\rho \ll \rho_{*}$ and $M_{\mathrm{d}}(R) \ll M_{*}(R)$, where $\rho_{*} / M_{*}(R)$ is the stellar central density/disk mass within a radius $R$ (dominating stellar disk mass);

2. $\rho \geq \rho_{*}$ and $0.5(H / R) M(R)<M_{\mathrm{d}}(R)<M(R)$, where $M(R)$ is the total mass enclosed within a radius $R$ (self-gravitating gas disk in $z$ direction).

In the following we call the model of a dominating stellar disk mass DSD model and the model of a self-gravitating gas disk in $z$ direction $S G Z$ model. The hydrostatic equilibrium in the vertical direction implies that the gravitational force is balanced by the turbulent pressure.

For the two cases the hydrostatic equilibrium has the following forms:

1. $p_{\text {turb }}=\Sigma \partial \Phi / \partial z$,

where $\Phi$ is the gravitational potential of the disk;

2. $p_{\text {turb }}=\pi G \Sigma^{2}$

(Paczyński 1978).

\subsection{Global gravitational stability in z direction}

The basic principles underlying the gravitational instability of a thin rotating disk can be found in Toomre (1964). A gaseous disk is locally stable to axisymmetric perturbations, if

$Q=\frac{v_{\text {turb }} \kappa}{\pi G \Sigma}>1$,

where $\kappa=\sqrt{R \frac{\mathrm{d} \Omega^{2}}{\mathrm{~d} R}+4 \Omega^{2}}$ is the epicylcic frequency. Since in general $\Omega \leq \kappa \leq 2 \Omega$, we will use the following equation:

$Q \simeq \frac{v_{\text {turb }} \Omega}{\pi G \Sigma}$. 
Multiplying the numerator and the denominator of the right hand side by $R^{2}$ gives

$$
Q=\frac{v_{\text {turb }}}{v_{\text {rot }}} \frac{M_{\text {tot }}}{M_{\text {gas }}},
$$

where $M_{\text {tot/gas }}$ is the total enclosed mass and the total enclosed gas mass at radius $R$. Thus for a given velocity dispersion $Q^{-1}$ is proportional to the ratio of gas mass to total mass.

\subsection{The star formation rate}

It is generally accepted that the star formation rate is proportional to the mean density of the disk and the inverse of the characteristic time scale for the cloud collapse, i.e. the nonaveraged local free fall time $t_{\mathrm{ff}}^{1}$ (see Sect. 3.1):

$\dot{\rho}_{*} \propto \frac{\rho}{t_{\mathrm{ff}}^{1}}$.

Since $t_{\mathrm{ff}}^{1} \propto \rho^{-\frac{1}{2}}$ (see Eq. (3)) this corresponds to a Schmidt law of the form $\dot{\rho}_{*} \propto \rho^{\frac{3}{2}}$. The factor of proportionality is given by the probability to find a self-gravitating cloud, i.e. the volume filling factor $\phi_{\mathrm{V}}$. Thus, the star formation rate is given by

$\dot{\rho}_{*}=\phi_{\mathrm{V}} \frac{\rho}{t_{\mathrm{ff}}^{\mathrm{l}}}=\sqrt{\phi_{\mathrm{V}}} \frac{\rho}{t_{\mathrm{ff}}^{\mathrm{H}}}$.

Furthermore, we assume that stars are only born in the midplane of the disk in regions that have the size of the turbulent driving length scale $l_{\text {driv }}$, because the clouds can collapse only within the turbulent time scale $t_{\text {turb }}=l_{\text {driv }} / v_{\text {turb }}$. We thus obtain

$\dot{\Sigma}_{*}=\dot{\rho}_{*} l_{\text {driv }}$

for the mass surface density turned into stars. The volume filling factor is defined such that $t_{1}=t_{\mathrm{ff}}^{1}=\delta^{-1} t_{\text {turb }}$ (Sect. 3.1). Combining Eq. (18) and Eq. (20) we get

$\dot{\Sigma}_{*}=\phi_{\mathrm{V}} \frac{\rho}{t_{\text {turb }}} \delta l_{\text {driv }}=\phi_{\mathrm{V}} \delta \rho v_{\text {turb }}$.

A simple estimate gives:

$t_{\text {turb }} \sim\left(\frac{l_{\text {driv }}}{100 \mathrm{pc}}\right)\left(\frac{v_{\text {turb }}}{10 \mathrm{~km} \mathrm{~s}^{-1}}\right)^{-1} \sim 10 \mathrm{Myr}$ and

$t_{\mathrm{ff}}^{1} \sim \sqrt{3 \pi /\left(32 G\left(\frac{\rho_{\mathrm{cl}}}{500 \mathrm{~cm}^{-3}}\right)\right)} \sim 2 \mathrm{Myr}$.

Since $t_{\text {turb }}=\delta t_{\mathrm{ff}}^{1}$, this leads to $\delta=5$, which is precisely the value we find in order to fit the gas properties of the Galaxy (Sect. 7).

\subsection{Energy flux conservation}

Here we assume that the energy input into the ISM due to SNe is transported practically without loss from the driving length scale to the dissipative length scale where it is radiated away. Mass is stored into self-gravitating clouds and eventually used for star formation. The energy per unit time which is transferred by turbulence is

$\dot{E} \simeq-\rho v \int v_{\text {turb }}^{2} / l_{\text {driv }}^{2} \mathrm{~d} V$ where the integration is taken over the volume $\int \mathrm{d} V=V=A H$ (Landau \& Lifschitz 1959). Thus, the energy flux per unit time and unit area is

$\frac{\Delta E}{\Delta t \Delta A}=-\Sigma v \frac{v_{\text {turb }}^{2}}{l_{\text {driv }}^{2}}$.

This energy loss is balanced by the SNe energy input

$\dot{E}_{\mathrm{SN}}+\dot{E}=0$.

We connect this energy input directly to the star formation rate (Eq. (20)). With the assumption of a constant initial mass function (IMF) independent of the environment one can write

$\frac{\dot{E}_{\mathrm{SN}}}{\Delta A}=\xi \dot{\Sigma}_{*}$

The factor of proportionality $\xi$ relates the local SN energy input to the local star formation rate and is taken to be independent of the radius. It can be normalized with Galactic observations by integrating over the Galactic disk: The number of SN per time is proportional to the Galactic integrated star formation rate:

$\dot{N}_{\mathrm{SN}}=\tilde{\xi} \dot{M}_{*}$.

The energy input per surface area and time in Eq. (25) is

$\frac{\Delta E}{\Delta A \Delta t}=\frac{E_{\mathrm{SN}}^{\mathrm{kin}} \dot{N}_{\mathrm{SN}}}{\Delta A}=\frac{E_{\mathrm{SN}}^{\mathrm{kin}} \tilde{\xi} \dot{M}_{*}}{\Delta A}=\tilde{\xi} E_{\mathrm{SN}}^{\mathrm{kin}} \dot{\Sigma}_{*}=\xi \dot{\Sigma}_{*}$,

where $E_{\mathrm{SN}}^{\mathrm{kin}}$ is the kinetic energy input from a single $\mathrm{SN}$. Thus the energy released into the ISM per mass turned into stars is

$\xi=\frac{\dot{N}_{\mathrm{SN}}}{\dot{M}_{*}} E_{\mathrm{SN}}^{\mathrm{kin}}$

Thornton et al. (1998) have shown by modeling SN explosions in different environments that the kinetic energy of the remnants is $\sim 10 \%$ of the total SN energy $E_{\mathrm{SN}}^{\text {tot }}$ irrespective of the density and metalicity of the ambient medium. The SN energy input into the ISM is thus $E_{\mathrm{SN}}^{\mathrm{kin}} \simeq 10^{50} \mathrm{ergs}$. The integrated number of SNe type II in the Galaxy is taken to be $\dot{N}_{\mathrm{SN}} \sim 1 / 40 \mathrm{yr}^{-1}$ in accordance with Tammann et al. (1994). The Galactic star formation rate is taken to be $\dot{M}_{*}=3 M_{\odot} \mathrm{yr}^{-1}$ (Prantzos \& Aubert 1995). This leads to $\xi \simeq 4.6 \times 10^{-8}(\mathrm{pc} / \mathrm{yr})^{2}$.

\subsection{The molecular fraction}

In order to derive an expression for the molecular fraction of gas in the disk, we compare the crossing time of the turbulent layer $t_{\text {turb }}$ and the $\mathrm{H}-\mathrm{H}_{2}$ transition time scale $t_{\mathrm{H}_{2}}=\alpha \phi_{\mathrm{V}} / \rho$ (Hollenbach \& Tielens 1997). We define the molecular fraction here as $f_{\text {mol }}=t_{\text {turb }} / t_{\mathrm{H}_{2}}$. This allows us to calculate the molecular surface density and total mass that can be compared with observations.

\section{Results}

With $\xi$ fixed and with a given rotation curve our model has only three free parameters: the constant of the molecule formation rate $\alpha$, the Toomre parameter $Q$ and the mass accretion 
rate within the disk $\dot{M}$. All disk properties can be expressed as functions of $\alpha, Q, \delta, \dot{M}, \xi, \Omega, \partial \Omega / \partial R$, and $R$. We have solved the set of Eqs. (8), (11), (12), (13)/(14), (16), and (26), for the two cases:

1. dominating stellar disk (DSD) $(Q>1, \mathrm{~S} 0$ and gas deficient galaxies);

2. self-gravitating gas disk in $z$ direction (SGZ) $(Q \sim 1$, spiral galaxies).

We will use $\Omega^{\prime}=\partial \Omega / \partial R$ with $\Omega^{\prime}<0$ in the region of interest.

\subsection{Dominating stellar disk (DSD)}

In this case the vertical pressure equilibrium is given by $p_{\text {grav }}=$ $\Sigma \partial \Phi / \partial z$. We make the approximation $\partial \Phi / \partial z \sim \chi v_{\text {rot }}^{2} R^{-1}$ leading to $p_{\text {grav }}=\chi \pi G \Sigma_{*} \Sigma$ (see e.g. Binney \& Tremaine 1987). The factor $\chi$ allows a smooth transition between the SGZ model (Sect. 4.2) and the DSD model.

As in Sect. 4.2 we first give the expression for $H, l_{\text {driv }}, \rho$, and $\Sigma$ as functions of $v_{\text {turb }}$.

$H=\chi^{-1} R\left(\frac{v_{\text {turb }}}{v_{\text {rot }}}\right)^{2}$

$l_{\text {driv }}=\sqrt{\frac{3}{2}} \frac{\pi}{8} \chi^{\frac{1}{2}} G \dot{M} Q^{\frac{3}{2}} v_{\text {turb }}^{-\frac{5}{2}} \Omega^{\frac{1}{2}} R^{-\frac{1}{2}}\left(-\Omega^{\prime}\right)^{-1}$,

$\rho=\pi^{-1} \chi G^{-1} \dot{M}^{-1} \Omega^{2} \frac{v_{\text {rot }}}{v_{\text {turb }}}$,

$\Sigma=\pi^{-1} G^{-1} Q^{-1} v_{\text {turb }} \Omega$.

The expression for the turbulent velocity dispersion is

$v_{\text {turb }}=0.87 \chi^{\frac{4}{15}} G^{\frac{1}{5}} \dot{M}^{\frac{1}{5}} Q^{\frac{4}{15}} \delta^{-\frac{1}{15}} \xi^{\frac{1}{15}} R^{\frac{1}{15}} \Omega^{\frac{7}{15}}\left(-\Omega^{\prime}\right)^{-\frac{1}{5}}$.

Inserting Eq. (35) into Eqs. (31), (32), and (34) leads to

$H=0.75 \chi^{-\frac{7}{15}} G^{\frac{2}{5}} \dot{M}^{\frac{2}{5}} Q^{\frac{8}{15}} \delta^{-\frac{2}{15}} \xi^{\frac{2}{15}} R^{-\frac{13}{15}} \Omega^{-\frac{16}{15}}\left(-\Omega^{\prime}\right)^{-\frac{2}{5}}$,

$l_{\text {driv }}=0.69 \chi^{-\frac{1}{6}} G^{\frac{1}{2}} \dot{M}^{\frac{1}{2}} Q^{\frac{5}{6}} \delta^{\frac{1}{6}} \xi^{-\frac{1}{6}} R^{-\frac{2}{3}} \Omega^{-\frac{2}{3}}\left(-\Omega^{\prime}\right)^{-\frac{1}{2}}$,

$\Sigma=0.28 \chi^{\frac{4}{15}} G^{-\frac{4}{5}} \dot{M}^{\frac{1}{5}} Q^{-\frac{11}{15}} \delta^{-\frac{1}{15}} \xi^{\frac{1}{15}} R^{\frac{1}{15}} \Omega^{\frac{22}{15}}\left(-\Omega^{\prime}\right)^{-\frac{1}{5}}$.

The viscosity $v$, the volume filling factor $\phi_{\mathrm{V}}$, the star formation rate $\dot{\Sigma}_{*}$, and the molecular fraction $f_{\text {mol }}$ then write

$v=0.58 \chi^{-\frac{4}{15}} G^{\frac{4}{5}} \dot{M}^{\frac{4}{5}} Q^{\frac{11}{15}} \delta^{\frac{1}{15}} \xi^{-\frac{1}{15}} R^{-\frac{16}{15}} \Omega^{-\frac{7}{15}}\left(-\Omega^{\prime}\right)^{-\frac{4}{5}}$,

$\phi_{\mathrm{V}}=0.79 \chi^{-\frac{2}{15}} G^{\frac{2}{5}} \dot{M}^{\frac{2}{5}} Q^{-\frac{2}{15}} \delta^{-\frac{22}{15}} \xi^{-\frac{8}{15}} R^{-\frac{8}{15}} \Omega^{\frac{4}{15}}\left(-\Omega^{\prime}\right)^{-\frac{2}{5}}$,

$\dot{\Sigma}_{*}=0.25 \chi^{\frac{13}{15}} G^{-\frac{3}{5}} \dot{M}^{\frac{2}{5}} Q^{-\frac{17}{15}} \delta^{-\frac{7}{15}} \xi^{-\frac{8}{15}} R^{\frac{7}{15}} \Omega^{\frac{49}{15}}\left(-\Omega^{\prime}\right)^{-\frac{2}{5}}$,

$f_{\mathrm{mol}}=0.37 \chi^{\frac{13}{30}} G^{-\frac{13}{10}} \dot{M}^{-\frac{3}{10}} Q^{-\frac{17}{30}} \xi^{\frac{7}{30}} R^{\frac{11}{15}} \Omega^{\frac{17}{15}}\left(-\Omega^{\prime}\right)^{\frac{3}{10}} \alpha^{-1}$.

Setting $\Omega^{\prime} \simeq-\Omega / R$ and $v_{\text {rot }}=\Omega R$ leads to the following simple radial dependences: $v_{\text {turb }}=\left(\chi v_{\text {rot }}\right)^{\frac{4}{15}}, H \propto\left(\chi v_{\text {rot }}\right)^{-\frac{7}{15}} \Omega^{-1}$, $l_{\text {driv }} \propto\left(\chi v_{\text {rot }}\right)^{-\frac{1}{6}} \Omega^{-1}, \Sigma \propto\left(\chi v_{\text {rot }}\right)^{\frac{4}{15}} \Omega, v \propto\left(\chi v_{\text {rot }}\right)^{-\frac{4}{15}} \Omega^{-1}$, $\phi_{\mathrm{V}}=\left(\chi v_{\text {rot }}\right)^{-\frac{2}{15}}, \dot{\Sigma}_{*} \propto\left(\chi v_{\text {rot }}\right)^{\frac{13}{15}} \Omega^{2}$, and $f_{\text {mol }} \propto\left(\chi v_{\text {rot }}\right)^{\frac{13}{30}} \Omega$.

We found that for $Q=1$ and $\chi \sim 0.05$ there is a smooth transition between the SGZ and DSD model, thus $\partial \Phi / \partial z \simeq 0.05 v_{\text {rot }}^{2} R^{-1}$. Figure 1 shows the radial dependences of the above parameters for (i) a constant rotation curve and (ii) for a rising rotation curve. We have included an arbitrary rising rotation curve to show the general effects of $\left(-\Omega^{\prime}\right)$ on the disk properties. We chose $\delta$ in a way to fit the observed volume filling factor of molecular clouds (Table 1). For this model $\chi=0.05, Q=10, \delta=5, \dot{M}=10^{-1} M_{\odot} \mathrm{yr}^{-1}$, and $\alpha=3 \times 10^{7} \mathrm{yr} M_{\odot} \mathrm{pc}^{-3}$. The rising rotation curve leads to a bigger driving length scale, disk height, viscosity, and volume filling factor. On the other hand, it leads to a smaller surface density, density, molecular fraction, and star formation rate in the inner disk than for a constant rotation curve. The total gas mass is $M_{\text {gas }} \sim 1.3 / 1.0 \times 10^{9} M_{\odot}$, the total molecular mass is $M_{\text {gas }} \sim 1.7 / 0.5 \times 10^{8} M_{\odot}$, and the total star formation is $\dot{M}_{*} \sim 0.2 / 0.1 M_{\odot} \mathrm{yr}^{-1}$ for the model with a flat and a rising rotation curve, respectively. This model applies to gas poor spiral and S0 galaxies.

For this model we have used $\dot{\Sigma}_{*}=\dot{\rho}_{*} l_{\text {driv }}$. This is only valid if $H / l_{\text {driv }}>1$ with

$\frac{H}{l_{\text {driv }}}=1.09 \chi^{-\frac{3}{10}} G^{-\frac{1}{10}} \dot{M}^{-\frac{1}{10}} Q^{-\frac{3}{10}} \delta^{-\frac{3}{10}} \xi^{\frac{3}{10}} v_{\text {rot }}^{-\frac{3}{10}}$.

For $v_{\text {rot }}=220 \mathrm{~km} \mathrm{~s}^{-1}, \dot{M}=10^{-2} M_{\odot} \mathrm{yr}^{-1}$, and $\chi=0.05$ this translates into the requirement $Q<1440$.

In the case of $H / l_{\text {driv }}<1$, we set $\dot{\Sigma}_{*}=\dot{\rho}_{*} H$. Whereas Eqs. (31)-(34) do not change, the turbulent velocity becomes

$v_{\text {turb }}=0.87 \chi^{\frac{5}{21}} G^{\frac{4}{21}} \dot{M}^{\frac{4}{21}} Q^{\frac{5}{21}} \delta^{-\frac{2}{21}} \xi^{\frac{2}{21}} R^{\frac{1}{21}} \Omega^{\frac{9}{21}}\left(-\Omega^{\prime}\right)^{-\frac{4}{21}}$.

The exponents are only slightly different from those of Eq. (35).

\subsection{Self-gravitating gas disk in z direction (SGZ)}

We first give the expressions for the disk height $H$, the turbulent driving length scale $l_{\text {driv }}$, the mean density in the disk plane $\rho$, and the disk surface density $\Sigma$ as functions of the Toomre parameter $Q$, the mass accretion rate $\dot{M}$, the angular velocity $\Omega$, the disk radius $R$, the radial derivative of the angular velocity $\Omega^{\prime}$, and the turbulent velocity dispersion $v_{\text {turb }}$.

$H=\frac{Q v_{\text {turb }}}{\Omega}$

$l_{\text {driv }}=\sqrt{\frac{3}{2}} \frac{\pi G \dot{M} Q}{8 R v_{\text {turb }}^{2}\left(-\Omega^{\prime}\right)}$

$\rho=\frac{\Omega^{2}}{\pi G Q^{2}}$

$\Sigma=\frac{v_{\text {turb }} \Omega}{\pi G Q}$

The expression for the turbulent velocity dispersion is

$v_{\text {turb }}=0.82 G^{\frac{3}{11}} \dot{M}^{\frac{3}{11}} \delta^{-\frac{1}{11}} \xi^{\frac{1}{11}} R^{-\frac{3}{11}} \Omega^{\frac{3}{11}}\left(-\Omega^{\prime}\right)^{-\frac{3}{11}}$.

Since $Q=\left(v_{\text {turb }} / v_{\text {rot }}\right)\left(M_{\text {tot }} / M_{\text {gas }}\right)($ Eq. (17)) and the turbulent velocity dispersion $v_{\text {turb }}$ is independent of $Q$, the Toomre parameter is only a measure of the ratio between the total enclosed and the total gas mass of the galaxy. 

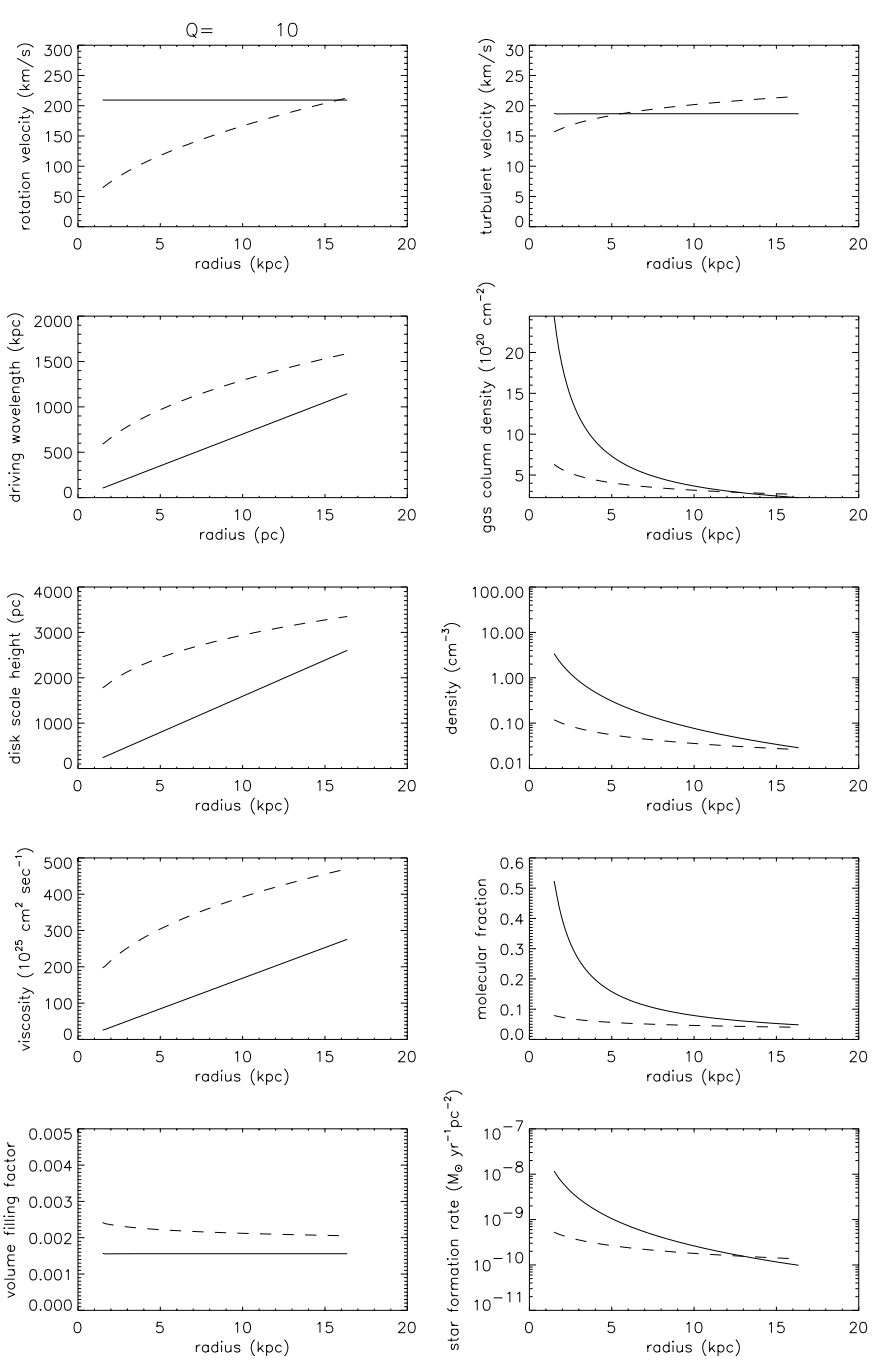

Fig. 1. Disk parameters for the case of the DSD model. Solid lines: constant rotation curve. Dashed line: rising rotation curve $v_{\text {rot }} \propto \sqrt{R}$.

Using Eq. (6) together with the pressure equilibrium in $z$ direction the viscosity intermittence factor $\gamma$ of Eq. (8) can be calculated

$\gamma=\frac{H}{l_{\text {driv }}} \sqrt{\phi_{\mathrm{V}}}=\sqrt{\frac{32}{3 \pi^{2}}} \simeq 1$.

Thus, in the SGZ model the viscosity reads

$v=\sqrt{\phi_{\mathrm{V}}} v_{\text {turb }} H=v_{\text {turb }} l_{\text {driv }}$.

Inserting Eq. (49) into Eqs. (45), (46), and (48) leads to

$$
\begin{aligned}
& H=0.82 G^{\frac{3}{11}} \dot{M}^{\frac{3}{11}} Q \delta^{-\frac{1}{11}} \xi^{\frac{1}{11}} R^{-\frac{3}{11}} \Omega^{-\frac{8}{11}}\left(-\Omega^{\prime}\right)^{-\frac{3}{11}}, \\
& l_{\text {driv }}=0.71 G^{\frac{5}{11}} \dot{M}^{\frac{5}{11}} Q \delta^{\frac{2}{11}} \xi^{-\frac{2}{11}} R^{-\frac{5}{11}} \Omega^{-\frac{6}{11}}\left(-\Omega^{\prime}\right)^{-\frac{5}{11}}, \\
& \Sigma=0.26 G^{-\frac{8}{11}} \dot{M}^{\frac{3}{11}} Q^{-1} \delta^{-\frac{1}{11}} \xi^{\frac{1}{11}} R^{-\frac{3}{11}} \Omega^{\frac{14}{11}}\left(-\Omega^{\prime}\right)^{-\frac{3}{11}}
\end{aligned}
$$

For the viscosity $v$, the volume filling factor $\phi_{\mathrm{V}}$, the star formation rate $\dot{\Sigma}_{*}$, and the molecular fraction $f_{\text {mol }}$ one obtains

$$
\begin{aligned}
& v=0.61 G^{\frac{8}{11}} \dot{M}^{\frac{8}{11}} Q \delta^{\frac{1}{11}} \xi^{-\frac{1}{11}} R^{-\frac{8}{11}} \Omega^{-\frac{3}{11}}\left(-\Omega^{\prime}\right)^{-\frac{8}{11}}, \\
& \phi_{\mathrm{V}}=0.81 G^{\frac{4}{11}} \dot{M}^{\frac{4}{11}} \delta^{-\frac{16}{11}} \xi^{-\frac{6}{11}} R^{-\frac{4}{11}} \Omega^{\frac{4}{11}}\left(-\Omega^{\prime}\right)^{-\frac{4}{11}},
\end{aligned}
$$

$\dot{\Sigma}_{*}=0.21 G^{-\frac{4}{11}} \dot{M}^{\frac{7}{11}} Q^{-2} \delta^{-\frac{6}{11}} \xi^{-\frac{5}{11}} R^{-\frac{7}{11}} \Omega^{\frac{29}{11}}\left(-\Omega^{\prime}\right)^{-\frac{7}{11}}$,

$f_{\text {mol }}=0.34 G^{-\frac{13}{11}} \dot{M}^{-\frac{2}{11}} Q^{-1} \xi^{\frac{3}{11}} R^{\frac{2}{11}} \Omega^{\frac{9}{11}}\left(-\Omega^{\prime}\right)^{\frac{2}{11}} \alpha^{-1}$.

Setting $\Omega^{\prime} \simeq-\Omega / R$ leads to the following simple radial scalings: $v_{\text {turb }}=$ const., $H \propto \Omega^{-1}, l_{\text {driv }} \propto \Omega^{-1}, \Sigma \propto \Omega, v \propto \Omega^{-1}$, $\phi_{\mathrm{V}}=$ const., $\dot{\Sigma}_{*} \propto \Omega^{2}$, and $f_{\text {mol }} \propto \Omega$.

Figure 2 shows the radial dependences of these parameters for (i) a constant rotation curve and (ii) for a rising rotation curve. We have again included a rising rotation curve to show the general effects of $\left(-\Omega^{\prime}\right)$ on the disk properties. For this model $Q=1, \delta=5, \dot{M}=10^{-1} M_{\odot} \mathrm{yr}^{-1}$, and $\alpha=3 \times 10^{7} \mathrm{yr}_{\odot} \mathrm{pc}^{-3}$ in order to fit Galactic observations. The rising rotation curve leads to a bigger driving length scale, disk height, viscosity, and volume filling factor. On the other hand, it leads to a smaller surface density, density, molecular fraction, and star formation rate mainly in the inner disk. The fraction between the gas density, and star formation rate for a constant and those for a rising rotation curve is about 5-10 in the central part of the galaxy. This fraction is about 3-5 for the surface density, driving length scale, disk height, and viscosity. The difference in the velocity dispersion and the volume filling factor is $20 \%$ and $30 \%$ respectively. The total gas mass for both models is $M_{\mathrm{gas}} \sim 6.8 \times 10^{9} M_{\odot}$, the total molecular mass is $M_{\text {mol }} \sim 3.0 \times 10^{9} M_{\odot}$, and the total star formation is $\dot{M}_{*} \sim 2.6 M_{\odot} \mathrm{yr}^{-1}$ in good agreement with Galactic observations (for the gas see, e.g. Kulkarni \& Heiles 1988; for the star formation rate see, e.g. Prantzos \& Aubert 1995).

\section{Comparison with the fully gravitational model of Vollmer \& Beckert (2002)}

In Vollmer \& Beckert (2002) we have analytically investigated the equilibrium state of a turbulent clumpy gas disk that consists of distinct self-gravitating clouds, which are embedded in a low density medium, and evolve in the fixed gravitational potential of the galaxy. Gravitational cloud-cloud interactions in the disk give rise to an effective viscosity and allows the transport of angular momentum and mass in the gas disk. In this scenario turbulence is assumed to be generated by instabilities involving self-gravitation and to be maintained by the energy input, which is provided by differential rotation of the disk and mass transfer to smaller galactic radii via cloud-cloud interactions. Both the energy source and dissipation process for turbulence in these disks are due to gravity. Therefore, we call this model the fully gravitational model.

\subsection{The viscosity prescription}

In the fully gravitational model the viscosity prescription is

$v=\operatorname{Re}^{-1} v_{\text {turb }} H$.

In Vollmer \& Beckert (2002) we have used a slightly different expression for the energy flux transferred by turbulence to smaller length scales: $\dot{E}=\rho v v_{\text {turb }}^{2} / l_{\text {driv }}=\Sigma v v_{\text {turb }}^{2} /\left(H l_{\text {driv }}\right)$ instead of $\dot{E}=\Sigma v v_{\text {turb }}^{2} / l_{\text {driv }}^{2}$. Thus we had already assumed $l_{\text {driv }}=H$. This lead to exponents of $Q$ that were twice that 

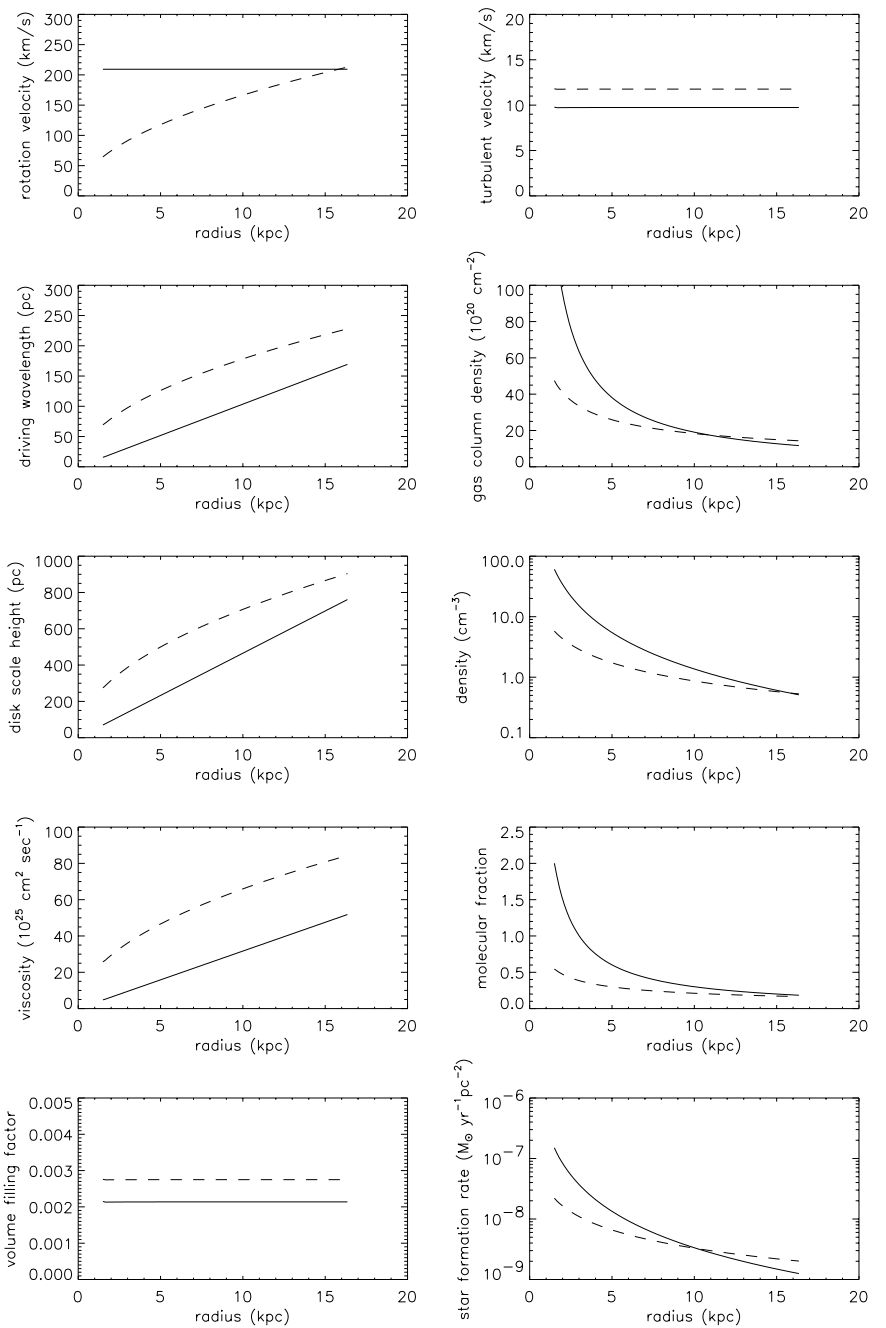

Fig. 2. Disk parameters for the SGZ model. Solid lines: disk with constant rotation curve. Dashed line: disk with rising rotation curve $v_{\text {rot }} \propto \sqrt{R}$.

of the model using Eq. (25). Therefore, the volume filling factor in the case of a turbulent non-Kolmogorov energy spectrum $(D=2)$ of the form $E(k) \propto k^{-2}$ in the framework of Vollmer \& Beckert (2002) using Eq. (25) is

$\phi_{\mathrm{V}} \simeq Q^{-2} R e^{-2}$.

For $Q=1$ one gets $\phi_{\mathrm{V}}=R e^{-2}$. In the present model including $\mathrm{SNe}$, Eq. (8) reads for the viscosity

$v=\sqrt{\phi_{\mathrm{V}}} v_{\text {turb }} \delta H$.

Thus, for $Q=1$ and $\delta=1$ the viscosity prescriptions of both models are equivalent.

\subsection{The star formation rate}

In Vollmer \& Beckert (2002) we suggested a star formation law of the form

$\dot{\Sigma}_{*}=R e^{-1} \Sigma \Omega$.

This has to be compared with the star formation rate in the present model, which reads (see Sect. 3.6).

$\dot{\Sigma}_{*}=\sqrt{\phi_{\mathrm{V}}} \rho l_{\text {driv }}\left(t_{\mathrm{ff}}^{\mathrm{H}}\right)^{-1}$.
In the case of the SGZ model $\rho=\left(\pi G Q^{2}\right)^{-1} \Omega^{2}$ (Eq. (47)). Thus, for $Q=1,\left(t_{\mathrm{ff}}^{\mathrm{H}}\right)^{-1} \simeq \Omega$ leading to a star formation rate of the form

$\dot{\Sigma}_{*}=\sqrt{\phi_{\mathrm{V}}} \rho l_{\text {driv }} \Omega$.

Since in the fully gravitational model $l_{\text {driv }}=H$, the prescription for the star formation rate of both models are equivalent for $Q=1$ disks.

\subsection{Energy flux conservation}

The main difference between the fully gravitational model and the model including $\mathrm{SNe}$ lies in the energy flux conservation equation. The energy transferred by turbulence to smaller length scales is balanced by the energy input through $\mathrm{SNe}$ in our present model, whereas it is balanced by the differential rotation and mass inflow in the fully gravitational model (Vollmer \& Beckert 2002).

\subsection{Results}

If one approximates $\Omega^{\prime} \sim-\Omega / R$, the radial scalings of all disk properties is the same for both SGZ models, i.e. $v_{\text {turb }}=$ const., $H \propto \Omega^{-1}, l_{\text {driv }} \propto \Omega^{-1}, \Sigma \propto \Omega, v \propto \Omega^{-1}, \phi_{\mathrm{V}}=$ const., $\dot{\Sigma}_{*} \propto \Omega^{2}$, and $f_{\text {mol }} \propto \Omega$.

We therefore conclude that the fully gravitational model (Vollmer \& Beckert 2002) and the here presented model including SNe lead to the same radial dependences of the disk properties in the case of $Q=1$ with $R e=\phi_{\mathrm{V}}^{-2}$. A quantitative comparison will be made in Sect. 7.

For the comparison with previous models of turbulent, selfgravitating gas disks we refer to Sect. 5.1 in Vollmer \& Beckert (2002).

\section{SN remnant size as driving wavelength (SNR)}

In Sect. 3.7 the energy flux conservation equation determines the turbulent driving wavelength $l_{\text {driv }}$. Alternatively, one can assume that only the interaction of SN bubbles leads to an effective turbulent viscosity that transports angular momentum. In this case the driving wavelength is twice the radius of the SN remnant at a time when the shock thermalizes. In the following we will call this model the SNR model. Following Dorfi (1993) the final radius of a SN remnant is given by

$R_{\mathrm{SNR}}=l_{\mathrm{SN}} E_{51}^{\frac{11}{35}} n_{0}^{-\frac{13}{35}} \mathrm{pc}$,

where $l_{\mathrm{SN}}$ is the characteristic radius for $n_{0}=1 \mathrm{~cm}^{-3}, E_{51}=$ $E /\left(10^{51} \mathrm{erg}\right)$, and $n_{0}$ is the average local density in $\mathrm{cm}^{-3}$. We set $l_{\mathrm{SN}}=64 \mathrm{pc}$ (Dorfi 1993) and replace Eq. (26) by $l_{\text {driv }}=2 R_{\mathrm{SNR}}$. This results in

$v_{\text {turb }}=0.56 G^{\frac{11}{35}} Q^{\frac{9}{70}} E_{51}^{-\frac{11}{70}} l_{\mathrm{SN}}^{-\frac{1}{2}} \dot{M}^{\frac{1}{2}} R^{-\frac{1}{2}} \Omega^{\frac{13}{35}}\left(-\Omega^{\prime}\right)^{-\frac{1}{2}}$,

$H=0.56 G^{\frac{11}{35}} Q^{\frac{79}{70}} E_{51}^{-\frac{11}{70}} l_{\mathrm{SN}}^{-\frac{1}{2}} \dot{M}^{\frac{1}{2}} R^{-\frac{1}{2}} \Omega^{-\frac{22}{35}}\left(-\Omega^{\prime}\right)^{-\frac{1}{2}}$,

$l_{\text {driv }}=1.53 G^{\frac{13}{35}} Q^{\frac{26}{35}} E_{51}^{\frac{11}{35}} l_{\mathrm{SN}} \Omega^{-\frac{26}{35}}$, 
$\rho=\frac{\Omega^{2}}{\pi G Q^{2}}$,

$\Sigma=0.18 G^{-\frac{24}{35}} Q^{-\frac{61}{70}} E_{51}^{-\frac{11}{70}} l_{\mathrm{SN}}^{-\frac{1}{2}} \dot{M}^{\frac{1}{2}} R^{-\frac{1}{2}} \Omega^{\frac{48}{35}}\left(-\Omega^{\prime}\right)^{-\frac{1}{2}}$,

$v=0.89 G^{\frac{24}{35}} Q^{\frac{61}{70}} E_{51}^{\frac{11}{70}} l_{\mathrm{SN}}^{\frac{1}{2}} \dot{M}^{\frac{1}{2}} R^{-\frac{1}{2}} \Omega^{-\frac{13}{35}}\left(-\Omega^{\prime}\right)^{-\frac{1}{2}}$,

$\Phi_{\mathrm{V}}=8.05 G^{\frac{4}{35}} Q^{-\frac{27}{35}} \delta^{-2} E_{51}^{\frac{33}{35}} l_{\mathrm{SN}}^{3} \dot{M}^{-1} R \Omega^{-\frac{8}{35}}\left(-\Omega^{\prime}\right)$,

$\dot{\Sigma}_{*}=1.44 G^{-\frac{4}{7}} Q^{-\frac{37}{14}} \delta^{-1} E_{51}^{\frac{11}{14}} \frac{5}{l_{\mathrm{S}}^{2}} \dot{M}^{-\frac{1}{2}} R^{\frac{1}{2}} \Omega^{\frac{15}{7}}\left(-\Omega^{\prime}\right)^{\frac{1}{2}}$,

$f_{\mathrm{mol}}=0.11 G^{-\frac{37}{35}} Q^{-\frac{43}{10}} \delta E_{51}^{-\frac{33}{71}} l_{\mathrm{SN}}^{-\frac{3}{2}} \dot{M}^{\frac{1}{2}} R^{-\frac{1}{2}} \Omega^{\frac{39}{35}}\left(-\Omega^{\prime}\right)^{-\frac{1}{2}} \alpha^{-1}$.

The intermittence factor $\gamma$ (Eq. (9)) is in this case $\gamma \sim 1$ as for the SGZ model.

Setting $\Omega^{\prime} \simeq-\Omega / R$ leads to the following radial scalings: $v_{\text {turb }} \propto \Omega^{-9 / 70}, H \propto \Omega^{-79 / 70}, l_{\text {driv }} \propto \Omega^{-26 / 35}, \Sigma \propto \Omega^{61 / 70}, v \propto$ $\Omega^{-61 / 70}, \Phi_{\mathrm{V}} \propto \Omega^{27 / 35}, \dot{\Sigma}_{*} \propto \Omega^{37 / 14}$, and $f_{\mathrm{mol}} \propto \Omega^{43 / 70}$. The exponents are quite close to those of the SGZ model.

We assume $\dot{M}=10^{-1} M_{\odot} \mathrm{yr}^{-1}, Q=1, \delta=5$, and $\alpha=2 \times$ $10^{7} \mathrm{yr} M_{\odot} \mathrm{pc}^{-3}$. Figure 3 shows these parameters for a constant and a rising rotation curve. In comparison to the SGZ model, the turbulent velocity dispersion and the volume filling factor are not constant with radius. For a constant rotation curve the latter increases by a factor 4 from the outer disk to the center of the Galaxy. All other quantities are similar to those of the SGZ model.

The total gas mass for both rotation curves is $M_{\text {gas }} \sim$ $6.3 \times 10^{9} M_{\odot}$ and the total molecular gas mass is $M_{\mathrm{mol}} \sim$ $2.0 \times 10^{8} M_{\odot}$. The total star formation rate is $\dot{M}_{*} \sim 0.8 M_{\odot} \mathrm{yr}^{-1}$ for the rising rotation curve and $\dot{M}_{*} \sim 5.7 M_{\odot} \mathrm{yr}^{-1}$ for the constant rotation curve.

The star formation rate for a constant rotation curve is a factor 2 higher than the observed Galactic value. Alternatively, if one drops Eq. (20) in the initial equations, one can deduce the star formation rate a posteriori with the help of the energy flux conservation equation (Eq. (26)):

$\dot{\Sigma}_{*}=\xi^{-1} \Sigma v\left(\frac{v_{\text {turb }}}{l_{\text {driv }}}\right)^{2}$

This is shown for the constant rotation curve as a dotted line in the panel of the star formation rate in Fig. 3. Since $\Sigma v \sim$ $\dot{M} /(3 \pi)$ and $v_{\text {turb }}=$ const. Eq. (75) yields $\dot{\Sigma}_{*} \propto l_{\text {driv }}^{-2}$. Now, with $l_{\text {driv }} \propto \rho^{-13 / 35}$ (Eq. (65)), $\rho \propto \Omega^{2}$ (Eq. (69)), and $\Sigma \propto \Omega^{61 / 70}$ one obtains $\dot{\Sigma}_{*} \propto \rho^{0.75} \propto \Omega^{1.5} \propto \Sigma^{1.7}$, which is the observed Schmidt law. In this case the characteristic timescale for star formation is no longer the free fall time but it is proportional to the viscous timescale. The total star formation rate in this case is $\dot{M}_{*} \sim 1.8 M_{\odot} \mathrm{yr}^{-1}$.

A possible remedy would be to use the star formation prescription of Eq. (75) and assume a smaller $\xi$, i.e. a smaller fraction of the total SN energy that goes into turbulent motions. With $4 \%$ of the total SN energy in the form of kinetic energy input as suggested by Dorfi (1993) would raise the total star formation rate to $\dot{M}_{*} \sim 3.3 M_{\odot} \mathrm{yr}^{-1}$.
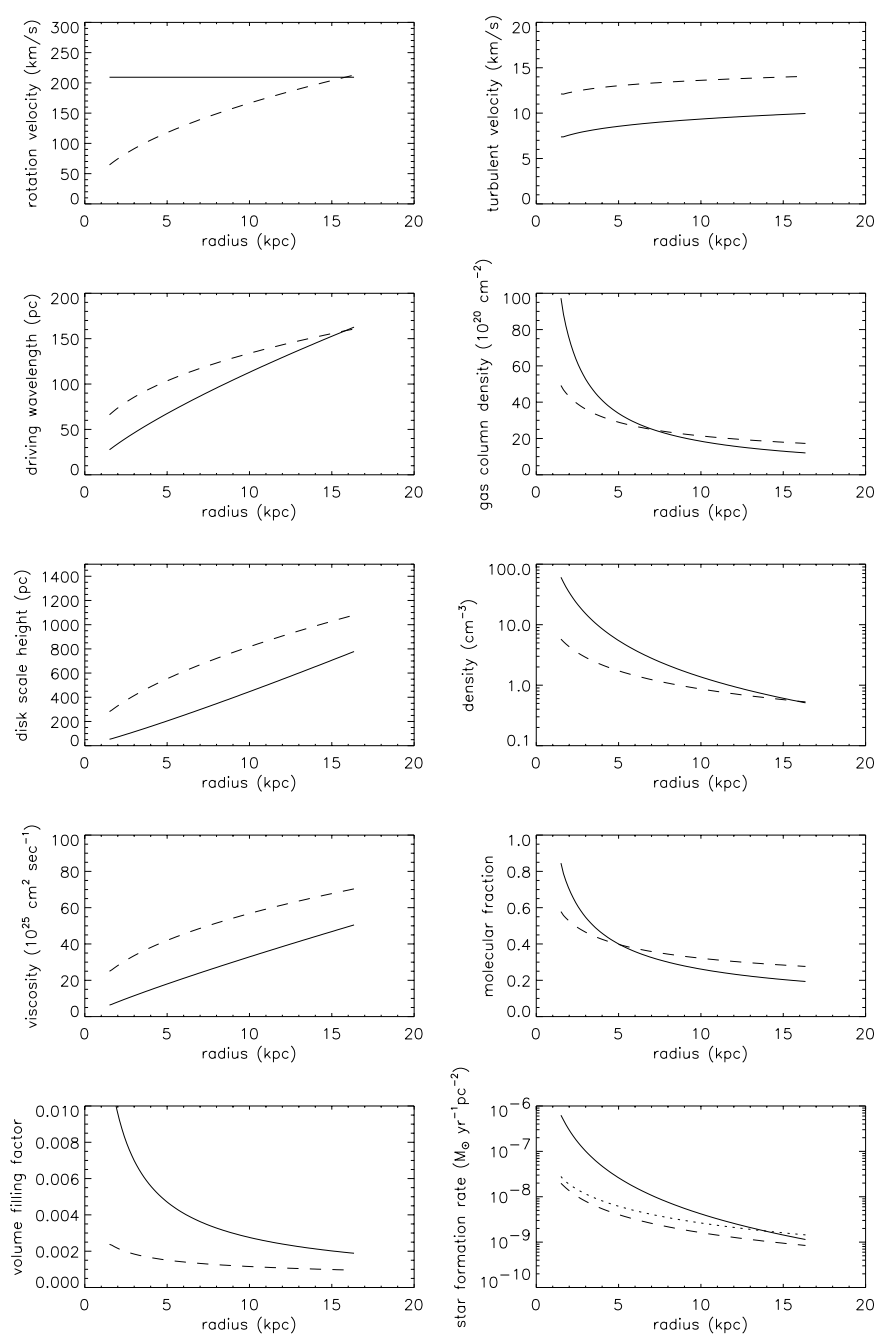

Fig. 3. Disk parameters for the case of SNR model. Solid lines: disk with constant rotation curve. Dashed line: disk with rising rotation curve $v_{\text {rot }} \propto \sqrt{R}$.

\section{Application to the Galaxy}

In this section we will investigate how close the simple analytical models of Sects. 4.2 and 6 can get to the observations of the Galaxy.

\subsection{Self-gravitating disk in $z$ direction (SGZ)}

We use $Q=1.0, \delta=5, \alpha=310^{7} \mathrm{yr}_{\odot} \mathrm{pc}^{-3}, \dot{M}=$ $10^{-1} M_{\odot} \mathrm{yr}^{-1}$, and $\xi=4.6 \times 10^{-8}(\mathrm{pc} / \mathrm{yr})^{2}$. Figure 4 shows the derived turbulent velocity, driving length scale, gas surface density, disk scale height, gas density, viscosity, molecular fraction, volume filling factor, and star formation rate for three different rotation velocities. For the model of the Galaxy we adopt $v_{\text {rot }}=220 \mathrm{~km} \mathrm{~s}^{-1}$. This results in a total gas mass of $M_{\text {gas }} \sim 6.8 \times 10^{9} M_{\odot}$, a total molecular mass of $M_{\text {mol }} \sim$ $3.3 \times 10^{9} M_{\odot}$, a total atomic gas mass of $M_{\mathrm{HI}} \sim 3.5 \times 10^{9} M_{\odot}$, and a total star formation rate of $\dot{M}_{*} \sim 2.6 M_{\odot} \mathrm{yr}^{-1}$.

The local density $\rho \sim 2 \mathrm{~cm}^{-3}$ and the local surface density of $\Sigma \sim 2 \times 10^{21} \mathrm{~cm}^{-2}$ at the solar radius (Binney \& Tremaine 1987) are well fitted by our model. At the solar radius the 
disk scale is $H \sim 400 \mathrm{pc}$, the turbulent driving scale length is $l_{\text {driv }} \sim 90 \mathrm{pc}$, and the effective viscosity is $v \sim 27 \times 10^{25} \mathrm{~cm}^{2} \mathrm{~s}^{-1}$. The driving wavelength is comparable to the generally accepted value of $50 \mathrm{pc} \leq l_{\text {driv }} \leq 150 \mathrm{pc}$ (see e.g. Ruzmaikin et al. 1988). Moreover, the derived star formation rate and gas surface density are comparable to those observed for the Galaxy.

The constant volume filling factor of $\phi_{\mathrm{V}} \sim 210^{-3}$ gives an equivalent Reynolds number of the fully gravitational model (Vollmer \& Beckert 2002) of $R e=22$ for a non-Kolmogorov spectrum $(D=2)$. In Vollmer $\&$ Beckert (2002) we used $Q=1$, $\dot{M}=10^{-2} M_{\odot} \mathrm{yr}^{-1}, \alpha=10^{7} \mathrm{yr} M_{\odot} \mathrm{pc}^{-3}$ and $R e=50$. All these parameters are within a factor 2 consistent with the values described above. This again shows the equivalence of the two models.

\section{2. $S N$ remnant size as driving length scale (SNR)}

For this model we use $Q=1, \delta=5, \alpha=210^{7} \mathrm{yr}_{\odot} \mathrm{pc}^{-3}$, $\dot{M}=10^{-1} M_{\odot} \mathrm{yr}^{-1}$, and $\xi=4.6 \times 10^{-8}(\mathrm{pc} / \mathrm{yr})^{2}$. The derived disk properties can be seen in Fig. 5. For a rotation velocity of $v_{\text {rot }}=220 \mathrm{~km} \mathrm{~s}^{-1}$, we obtain the following results: total gas mass $M_{\text {gas }} \sim 6.3 \times 10^{9} M_{\odot}$, total molecular mass of $M_{\text {mol }} \sim$ $2.0 \times 10^{9} M_{\odot}$, a total atomic gas mass of $M_{\mathrm{HI}} \sim 4.3 \times 10^{9} M_{\odot}$, and the total star formation rate given in Sect. 6.

The driving wavelength at the solar radius is $l_{\text {driv }}=100 \mathrm{pc}$. The other local quantities $(\Sigma, \rho, H)$ are comparable to those of Sect. 7.1. As already mentioned the star formation rate calculated using Eq. (20) is somewhat too high and that calculated using the energy flux conservation (Eq. (75)) is somewhat too low compared to the observed value.

\section{A realistic gravitational potential}

In this section we use an analytic gravitational potential $\Phi_{\mathrm{g}}$ given by Allen \& Santillán (1991). It consists of a disk, bulge, and halo component. We use the following values for the parameters of Allen \& Santillán (1991):

$$
\begin{aligned}
& \text { - bulge: } M_{1}=1.41 \times 10^{10} M_{\odot}, b_{1}=387 \mathrm{pc}, \\
& \text { - disk: } M_{2}=8.56 \times 10^{10} M_{\odot}, a_{2}=5318 \mathrm{pc}, b_{2}=250 \mathrm{pc}, \\
& \text { - halo: } M_{1}=1.07 \times 10^{11} M_{\odot}, a_{3}=12 \mathrm{kpc} .
\end{aligned}
$$

Vertical pressure equilibrium is described by

$\rho v_{\text {turb }}^{2}=\Sigma\left(\pi G \Sigma+\frac{\partial \Phi_{\mathrm{g}}}{\partial z}\right)$.

\subsection{Self-gravitating disk in $z$ direction}

We adopt $Q=1.0, \delta=5, \dot{M}=5 \times 10^{-2} M_{\odot} \mathrm{yr}^{-1}$, and $\alpha=$ $4 \times 10^{7} \mathrm{yr} M_{\odot} \mathrm{pc}^{-3}$.

The resulting radial profiles for the disk parameters are shown in Fig. 6. It turns out that the gas disk is close to the stage of self-gravitation in $z$ direction, but is still dominated by the gravitational potential of disk, bulge, and halo. The total gas mass is $M_{\mathrm{tot}}=7.5 \times 10^{9} M_{\odot}$, the total molecular mass is $M_{\mathrm{mol}}=2.8 \times 10^{9} M_{\odot}$, and the star formation rate is $\dot{M}_{*}=5.0 M_{\odot} \mathrm{yr}^{-1}$.
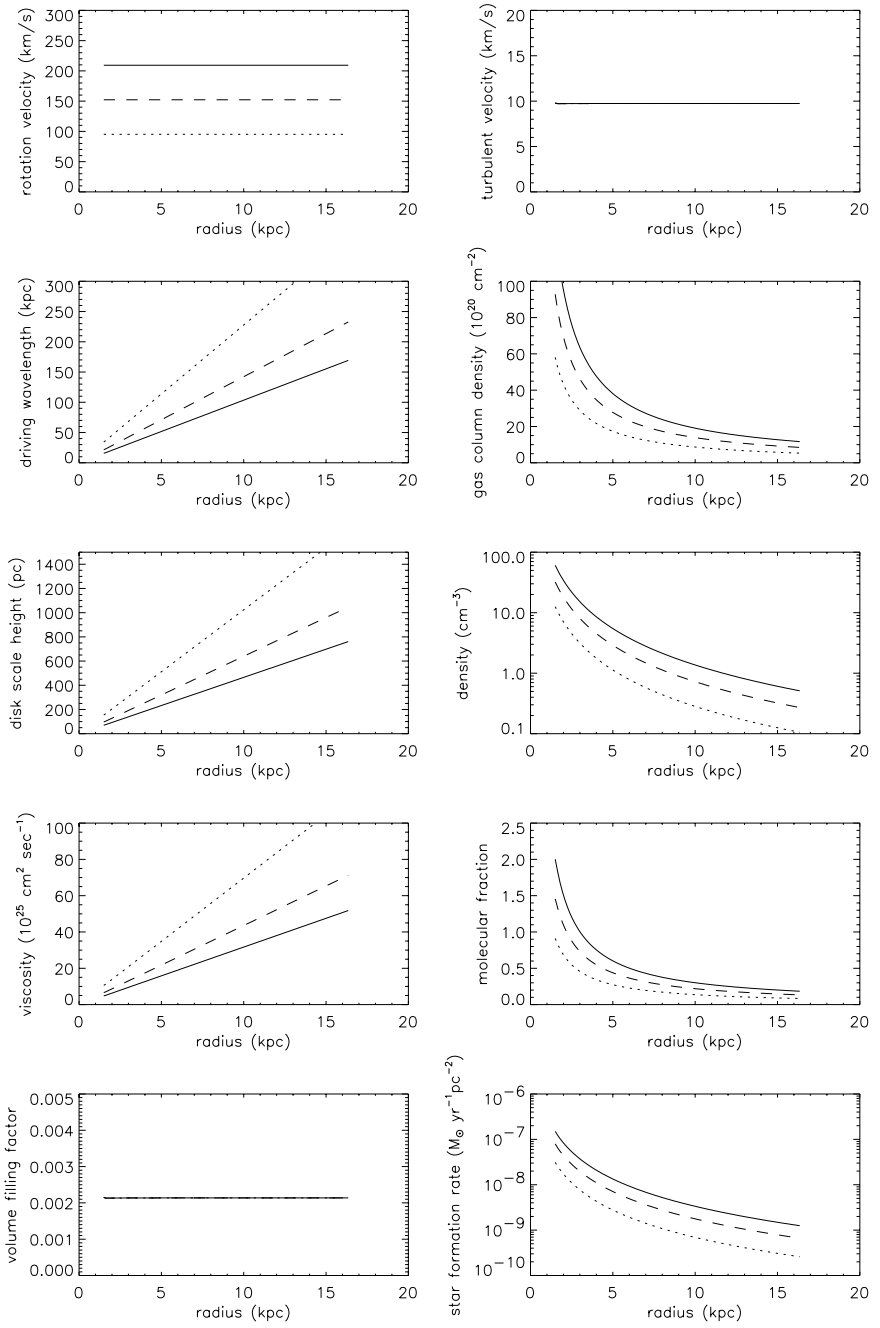

Fig. 4. Radial profiles of disk parameters for the case of the SGZ model for three different rotation velocities (solid lines: $v_{\text {rot }}=250 \mathrm{~km} \mathrm{~s}^{-1}$, dashed lines: $175 \mathrm{~km} \mathrm{~s}^{-1}$, dotted lines: $100 \mathrm{~km} \mathrm{~s}^{-1}$ ).

For $R>5 \mathrm{kpc}$ the gas surface density has approximately a $1 / R$ profile. The disk is flaring with an increasing flaring angle for increasing $R$. The volume filling factor is still approximately constant with radius. The star formation rate can be described by an exponential with a scale length of $\sim 3.5 \mathrm{kpc}$ for radii between 3 and $10 \mathrm{kpc}$.

\section{2. $S N$ remnant size as driving length scale (SNR)}

In the case of the SNR model we adopt slightly different parameters in order to match Galactic observations: $Q=1.0, \delta=5$, $\dot{M}=5 \times 10^{-2} M_{\odot} \mathrm{yr}^{-1}$, and $\alpha=2 \times 10^{7} \mathrm{yr}_{\odot} \mathrm{pc}^{-3}$. The resulting radial profiles for the disk parameters are shown in Fig. 7. The total gas mass is $M_{\text {tot }}=6.5 \times 10^{9} M_{\odot}$, the total molecular mass is $M_{\text {mol }}=2.7 \times 10^{9} M_{\odot}$, and the star formation rate is $\dot{M}_{*}=6.5 M_{\odot} \mathrm{yr}^{-1}$. All disk properties have values comparable to those of the SGZ model. The main differences to the SGZ model are that (i) the volume filling factor increases significantly with decreasing galactic radius, (ii) the star formation rate rises more steeply to the galaxy center, and (iii) the molecular fraction rises less steeply to the galaxy center than for the 

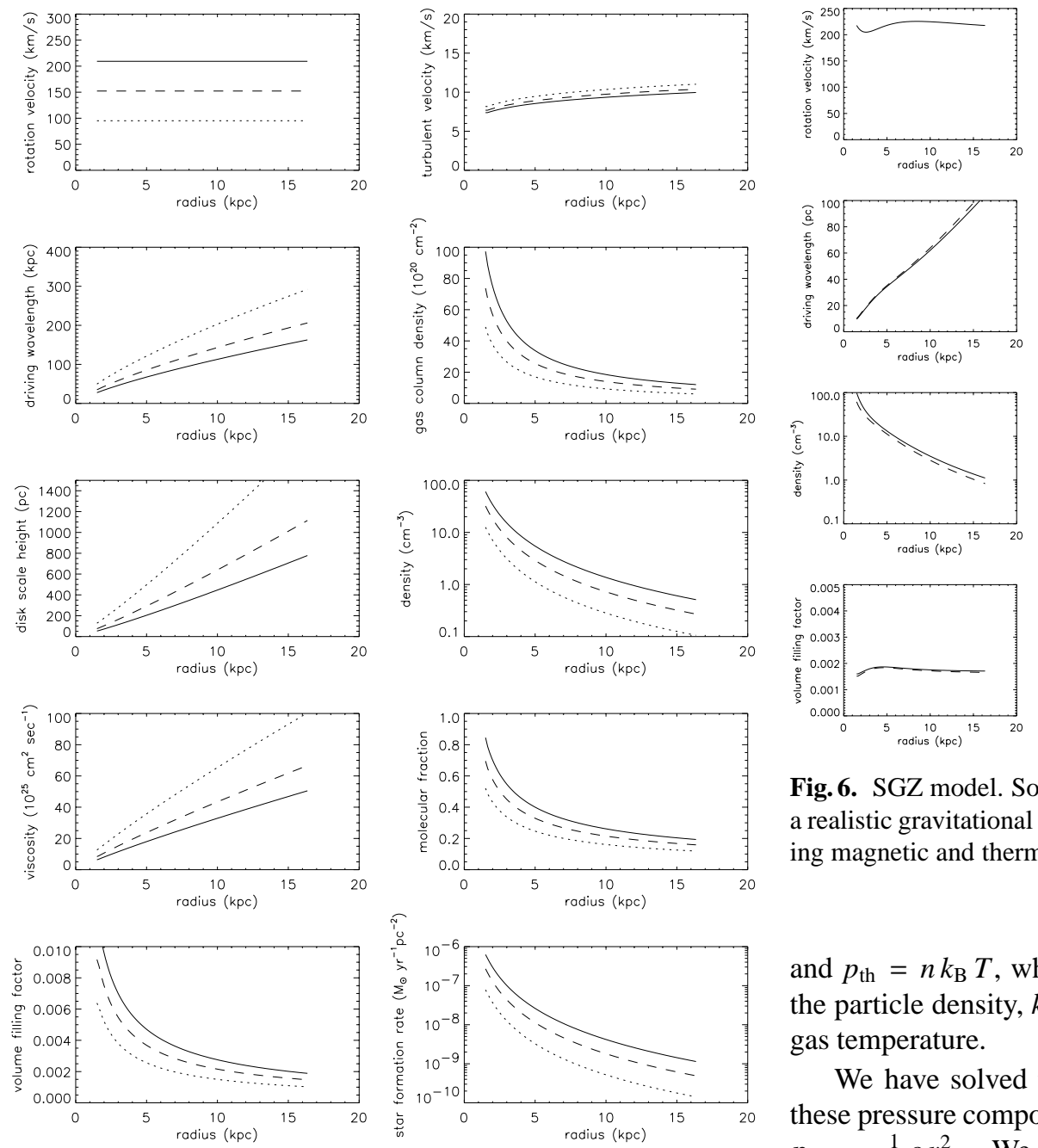

Fig. 5. Radial profiles of disk parameters for the case of the SNR model for three different rotation velocities (solid lines: $v_{\text {rot }}=$ $250 \mathrm{~km} \mathrm{~s}^{-1}$, dashed lines: $175 \mathrm{~km} \mathrm{~s}^{-1}$, dotted lines: $100 \mathrm{~km} \mathrm{~s}^{-1}$ ).

SGZ model. As for the SGZ model the gas surface density has approximately a $1 / R$ profile for $R>5 \mathrm{kpc}$. The star formation rate shows approximately a $1 / R^{2}$ profile.

\subsection{The HI to CO surface density relation}

Figure 8 shows the radial profiles of the total, molecular, and atomic gas surface density for the SGZ model. These profiles well resemble those found observationally by Wong \& Blitz (2002). The authors give the following relation between the atomic and the molecular gas surface density: $\Sigma_{\mathrm{HI}} / \Sigma_{\mathrm{CO}} \propto R^{1.5}$. In order to check our model for this relation we show in Fig. 9 the radial dependence of the ratio $\Sigma_{\mathrm{HI}} / \Sigma_{\mathrm{CO}}$ and its slope for the model SGZ. The curve for the SNR model has approximately the same slope. For $R>3 \mathrm{kpc}$ both models reproduce the observed exponent of 1.5 .

\subsection{Magnetic fields and thermal pressure}

For the vertical pressure equilibrium (Eqs. (13), (14)) we have neglected the magnetic and thermal pressure: $p_{\text {magn }}=B^{2} /(8 \pi)$
Fig. 6. SGZ model. Solid lines: radial profiles of disk parameters for a realistic gravitational potential. Dashed lines: disk properties including magnetic and thermal pressure.

and $p_{\text {th }}=n k_{\mathrm{B}} T$, where $B$ is the magnetic field strength, $n$ is the particle density, $k_{\mathrm{B}}$ is the Boltzmann constant and $T$ is the gas temperature.

We have solved the set of equations of Sect. 8 including these pressure components and setting the turbulent pressure to $p_{\text {turb }}=\frac{1}{2} \rho v_{\text {turb }}^{2}$. We assume equipartition between the energy density of the turbulent gas and the magnetic field $\frac{1}{2} \rho v_{\text {turb }}^{2}=$ $B^{2} /(8 \pi)$ and $T=10000 \mathrm{~K}$. The resulting disk properties are plotted as dashed lines in Figs. 6 and 7.

The magnetic field derived from equipartition decreases from $\sim 20 \mu \mathrm{G}$ in the center to $\sim 1 \mu \mathrm{G}$ at the edge of the disk. Its value at $8.5 \mathrm{kpc}$ is $\sim 7 \mu \mathrm{G}$. Due to the additional pressure terms the turbulent velocity, gas column density, gas density, molecular fraction, and the star formation rate decrease, whereas the driving length scale, disk scale height, and the viscosity increase. The volume filling factor decreases slightly in the case of the SGZ model and increases in the case of the SNR model.

The star formation rates are $\dot{M}_{*}=3.6 M_{\odot} \mathrm{yr}^{-1}$ for the SGZ model and $\dot{M}_{*}=4.2 M_{\odot} \mathrm{yr}^{-1}$ for the SNR model. The radial profile of the magnetic field then fits nicely that proposed by Strong et al. (2000) in its absolute values. It can be described by an exponential with a scale length of $8 \mathrm{kpc}$ and a local value of the magnetic field of $\sim 7 \mu \mathrm{G}$ for both models.

\subsection{The star formation law}

For the relation between the star formation and the total gas density, Wong \& Blitz (2002) used a Schmidt law of the form

$\dot{\Sigma}_{*} \propto \Sigma^{\beta}$. 

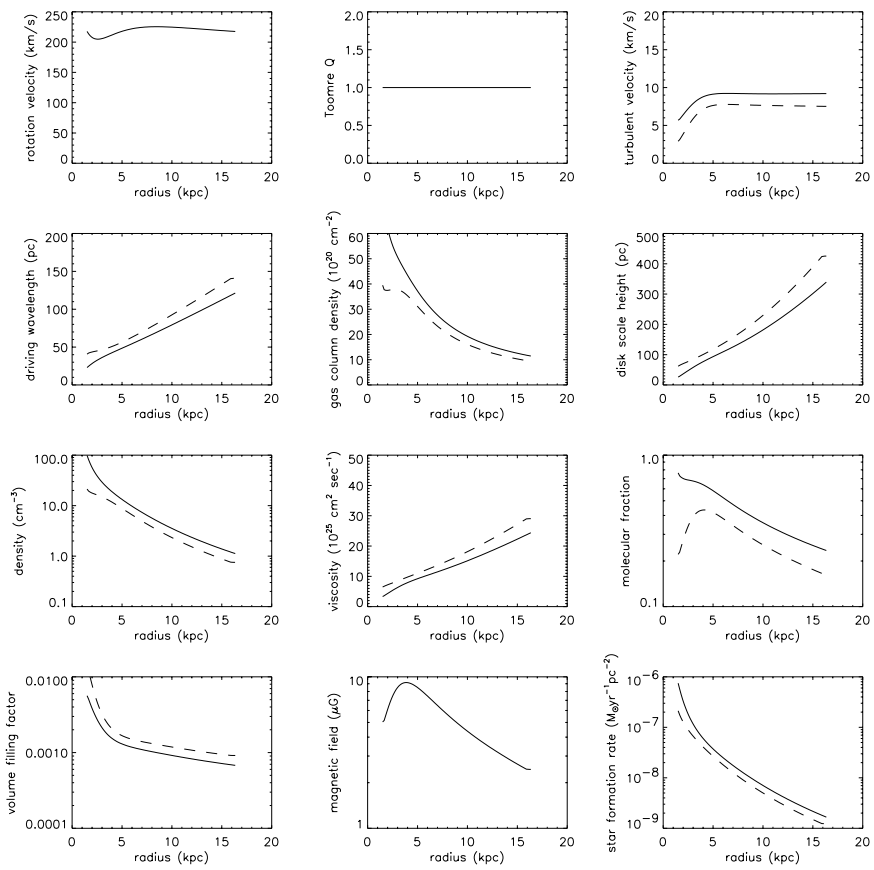

Fig. 7. SNR model. Solid lines: radial profiles of disk parameters for a realistic gravitational potential. Dashed lines: disk properties including magnetic and thermal pressure.

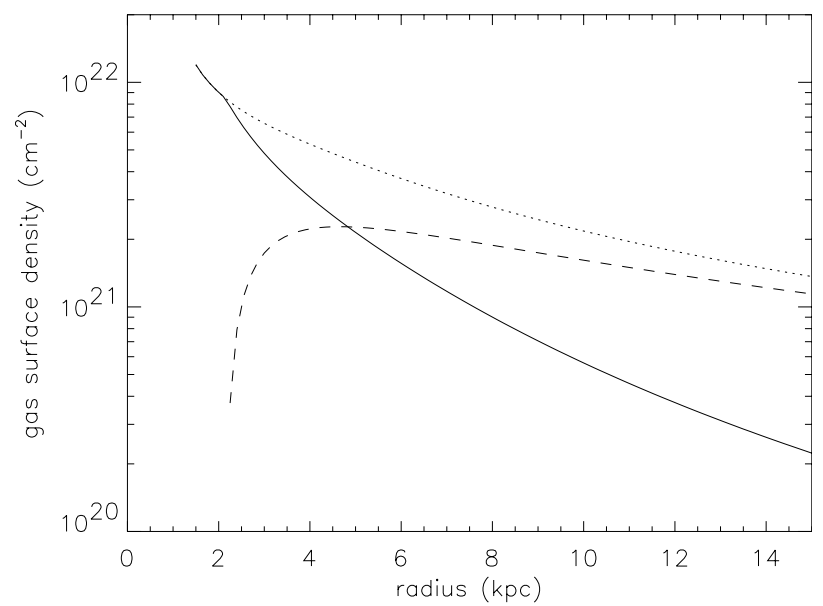

Fig. 8. Radial profiles of the total (dotted line), molecular (solid line), and atomic (dashed line) gas surface density.

They found $\beta=1.7 \pm 0.3$ if they use the star formation derived from $\mathrm{H} \alpha$ measurements corrected by an extinction that is proportional to $\Sigma$. Figure 10 shows the star formation rate $\dot{\Sigma}_{*}$ as a function of the total gas surface density $\Sigma$ for our model together with two curves for $\beta=1.5$ and $\beta=2$. The star formation rate of the SGZ model has an exponent $\beta=2$ for $\Sigma<50 M_{\odot} \mathrm{pc}^{-2}$ and $\beta=1.5$ for $\Sigma>50 M_{\odot} \mathrm{pc}^{-2}$. The star formation rate of the SNR model has always an exponent greater than 2 and steepens considerably for $\Sigma>50 M_{\odot} \mathrm{pc}^{-2}$. Thus, only the SGZ model can reproduce the observational results of Wong \& Blitz (2002).
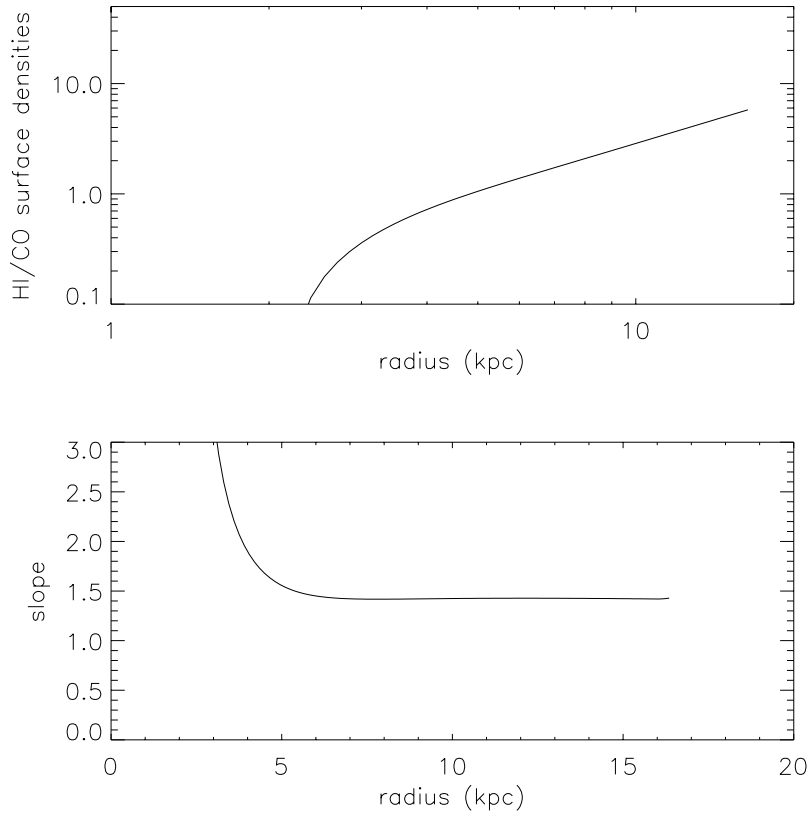

Fig. 9. The radial dependence of the ratio between the atomic and the molecular gas surface density $\Sigma_{\mathrm{HI}} / \Sigma_{\mathrm{CO}}$ (upper graph) and its slope (lower graph).

\section{Discussion}

The dominating stellar disk (DSD) model applies for gas deficient galaxies ( $\mathrm{S} 0$ and cluster galaxies). These are separate cases and will be investigated in more detail in a separate paper.

The model of a self-gravitating gas disk (SGZ model) and the model where the SN remnant size is assumed to be the driving length (SNR model) can be successfully applied to the Galaxy. The input parameters are $Q \sim 1, \delta=5, \dot{M} \sim$ $0.05-0.1 M_{\odot} \mathrm{yr}^{-1}, \xi \sim 4.6 \times 10^{-8}(\mathrm{pc} / \mathrm{yr})^{2}$, and $\alpha \sim 2-3 \times$ $10^{7} \mathrm{yr}_{\odot} \mathrm{pc}^{-3}$. The constants of molecule formation $\alpha$ in all models are very close to that given by Hollenbach \& Tielens (1997). The fully analytical models (Sects. 4.2 and 6) both describe the gas properties and the star formation rate of the Galaxy well. Their radial dependencies are very similar.

The models with a realistic gravitational potential fit observations well and lead to star formation rates that are consistent with the observed value. The star formation rate in the inner part of the galactic disk is more realistic for the SGZ than for the SNR model. Moreover, only the SGZ model reproduces the observed exponential radial profile of the star formation rate with the right length scale. Thus, the fit of the radial profile of the star formation rate favors the SGZ model.

Including a magnetic field in equipartition with the turbulent energy density leads to a realistic radial profile of the magnetic field in accordance with derived profiles in the literature.

The main difference between the two models is found in the radial profile of the volume filling factor of self-gravitating clouds, which is not well constrained by observations. Blitz (1993) states that a "glaring deficiency in galactic studies of giant molecular clouds is a quantitative study" of the radial dependence of their properties. Thus, both models can be regarded as equally valid. 


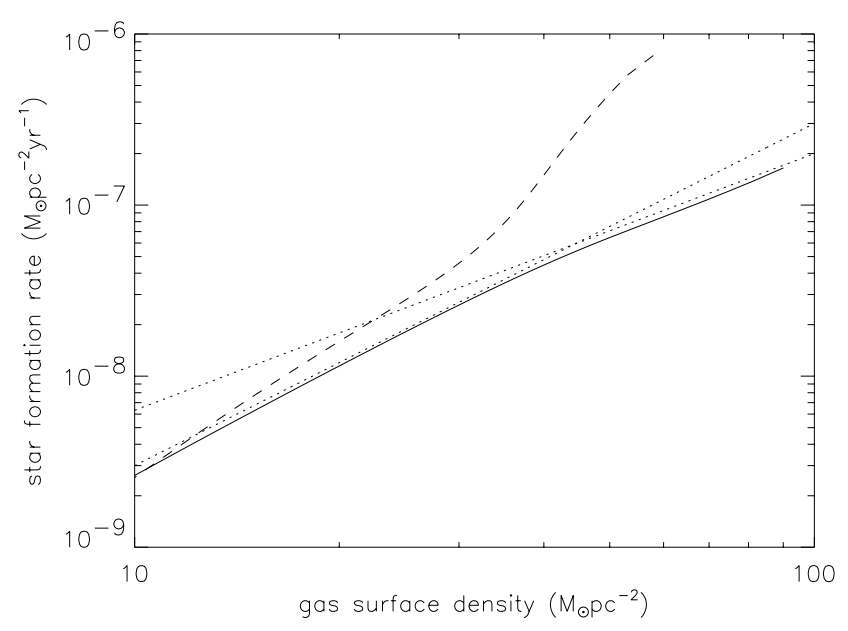

Fig. 10. The star formation rate $\dot{\Sigma}_{*}$ as a function of the total gas surface density $\Sigma$. Solid line: SGZ model. Dashed line: SNR model. Dotted lines: $\dot{\Sigma}_{*} \propto \Sigma^{1.5}$ and $\dot{\Sigma}_{*} \propto \Sigma^{2}$.

We conclude that our models correctly reproduce the following observations:

- the integrated total, molecular, and atomic gas mass;

- the integrated star formation rate within a factor of 2;

- the radial dependence of the ratio between the atomic and molecular gas surface densities.

The exponent for the Schmidt law might be $\sim 20 \%$ too high. In Sect. 5 we show that the viscosity and star formation prescriptions for the SN driven and the gravity-driven turbulence (without $\mathrm{SN}$ feedback) are equivalent for $Q=1$ and $\delta=1$. However, the absolute values of the energy flux $\frac{\Delta E}{\Delta t \Delta A}=-\Sigma v \frac{v_{\text {turb }}^{2}}{l_{\text {driv }}^{2}}$ (Eq. (25)) is different for the two models. In the case of gravitydriven turbulence the energy flux conservation equation together with the angular momentum equation and the vertical pressure equilibrium lead to $l_{\text {driv }}=H$ independent of a specific viscosity prescription. For the viscosity $v=\gamma v_{\text {turb }} l_{\text {driv }}$ (Eq. (5)) the gravity-driven turbulence model reads $\gamma=R e^{-1}$ (Eq. (59)) and $H / l_{\text {driv }}=1$, whereas the SGZ model gives $\gamma=1$ and $H / l_{\text {driv }}=4.5$. Thus the energy flux due to $\mathrm{SN}$ is a factor of $\sim 200$ larger than that due to gravitational instabilities. This implies that whenever the star formation exceeds $1 \%$ of its equilibrium value for a given galactic gas disk (see Sect. 4.2), the energy input due to $\mathrm{SN}$ dominates over that due to gravitational instabilities. Whenever it is smaller than $1 \%$ of its equilibrium value, gravitational instabilities take over and drive the ISM turbulence. In this case the mass accretion rate $\dot{M}$ is $\sim 10$ times smaller than that of the $\mathrm{SN}$ driven turbulent gas disk.

\section{Star formation}

In this section we study the behaviour of the star formation efficiency $(S F E)$ defined as the inverse of the star formation time scale:

$S F E=\left(t_{*}\right)^{-1}=\frac{\dot{\Sigma}_{*}}{\Sigma}$.

We are especially interested in the dependence of the $S F E$ on $Q$.

\subsection{Starbursts $(Q<1)$}

There are two possible definitions of a starburst:

1. Definition based on the absolute value of the star formation rate measured by the $\mathrm{H} \alpha$, radio continuum, or FIR. In this case, the star formation is enhanced, because of an enhanced gas surface density, e.g. the Schmidt law $\left(\dot{\Sigma}_{*} \propto \Sigma^{\beta}\right)$ is still valid.

2. Definition based on the $S F E$. In this case the $S F E$ is enhanced (the star formation time scale is reduced).

Rownd \& Young (1999) studied a sample of starburst galaxies. Out of 85 galaxies only 24 are starburst galaxies according to definition 2. Wong \& Blitz (2000) observed one of them, NGC 4736. They found, in agreement with Rownd \& Young (1999), that the nuclear $\mathrm{H} \alpha$ ring does not follow a Schmidt law, but has a much higher $S F E$ than expected by a Schmidt law.

Our SGZ model yields

$S F E=\frac{\dot{\Sigma_{*}}}{\Sigma} \propto Q^{-1}$

for the SGZ model and

$S F E=\frac{\dot{\Sigma}_{*}}{\Sigma} \propto Q^{-1.77}$

for the SNR model. In Sect. 4.2 we have shown that for the SGZ model the turbulent velocity dispersion is independent of $Q$ (Eq. (49)) and thus $Q$ measures the ratio between the total enclosed mass and the total gas mass, so that for a given galactic potential $Q \propto \Sigma^{-1}$. This is also approximately valid for the SNR model.

If the infall time scale is short enough that a considerable fraction of gas can be transported to small galactic radii where it is accumulated, star formation will be enhanced much stronger than predicted by the Schmidt law in this region. This is one possibility to create a central starburst. The massive infall needed might be due to a tidal interaction with another galaxy, an interaction between the ISM and the intracluster medium in a galaxy cluster, or a bar instability. Based on Eqs. (54) and (57) our model would then predict

$\dot{\Sigma}_{*} \propto \Sigma^{3}$.

However, one has to keep in mind that a $Q<1$ disk is not a stable configuration and can in principle not be described by an equilibrium disk. One has to solve the time dependent equation of transport of angular momentum. Thus, this estimate must be regarded with caution. Our equilibrium model suggests that at the beginning of a starburst the turbulent velocity stays constant at $10 \mathrm{~km} \mathrm{~s}^{-1}$, whereas the driving length scale is reduced. This counterbalances the enhanced star formation rate. Evidently, after a short time the available gas will be consumed and the SNe might heat the disk and lead rapidly to a $Q>1$ disk.

\subsection{Exhausted star formation $(Q>1)$}

In the opposite case, when gas is taken away from the galaxy by enhanced star formation or external effects, $Q$ becomes greater than unity. Then the DSD model applies giving

$\mathrm{SFE}=\frac{\dot{\Sigma}_{*}}{\Sigma} \propto Q^{-\frac{2}{5}}$ 
for the SGZ model. In this case star formation becomes less efficient, but this effect is not as pronounced as the increase for $Q<1$ discussed above in Sect. 10.1.

\section{Summary and conclusions}

We extend the model of a turbulent cloudy gas disk (Vollmer $\&$ Beckert 2002) by including energy input through SN explosions. This is realized (i) by adapting the energy flux conservation equation where the energy flux transferred by turbulence to smaller length scales is balanced by the energy input due to SN explosions (SGZ model) or alternatively (ii) by assuming that the size of a $\mathrm{SN}$ remnant equals the turbulent driving length scale (SNR model). In the SGZ model the SN energy flux is assumed to be proportional to the local star formation rate. The local star formation rate $\dot{\rho}_{*}$ is assumed to be proportional to the mean density and inversely proportional to the local free fall time of the clouds. The factor of proportionality is the probability to find a self-gravitating cloud, i.e. the volume filling factor. The integration length in $z$ direction is assumed to be the turbulent driving length scale, i.e. the length scale over which clouds are self-gravitating: $\dot{\Sigma}_{*}=\dot{\rho}_{*} l_{\text {driv }}$.

We assume the turbulence to be intermittent due to selfgravity. The viscosity $v$ is thus reduced to $v=\gamma v_{\text {turb }} l_{\text {driv }}$ with $\gamma \leq 1$. For $Q \leq 1$ we find $\gamma=1$.

For model (i) we have calculated two separate cases:

- a dominating stellar disk mass (DSD) $(Q>1)$ and

- a self-gravitating gas disk in $z$ direction (SGZ) $(Q \sim 1)$.

For the SGZ model and $\Omega^{\prime} \sim-\Omega / R$, the radial dependences of the disk properties (height, turbulent driving length scale, surface density, density, viscosity, volume filling factor, star formation rate, and molecular fraction) can be expressed as functions of the mass accretion rate $\dot{M}, Q$, the cloud size divided by the driving length scale $\delta$, the fraction of SN energy that is transformed into kinetic energy $\xi$, the constant of molecule formation $\alpha$, and the rotation velocity $\Omega(R)$. In the case of the DSD model, the dependences on $\Omega(R)$ are the same as for the SGZ model, whereas those on $\dot{M}, Q, \alpha$, and $\xi$ are different. In addition, a factor that depends on the rotation velocity $v_{\text {rot }}$ appears.

The SNR model gives radial dependences of the disk properties with exponents that are close to those of the SGZ model. Both analytical models (SGZ and SNR) reproduce an almost constant turbulent gas velocity as observed.

For the SGZ model we conclude that

1. the ratio between the turbulent driving length scale $l_{\text {driv }}$ and the disk height $H$ equals the square root of the volume filling factor $\phi_{\mathrm{V}}$,

2. for $Q=1$ the viscosity and star formation prescriptions are equivalent to those given for the fully gravitational model (Vollmer \& Beckert 2002),

3. the radial dependences are the same as those for the fully gravitational model.

The SGZ and SNR models are applied to the Galaxy and give a good description of its gas disk. We derive $Q \sim 1$ and a mass accretion rate within the disk of $\dot{M} \sim 0.05-0.1 M_{\odot} \mathrm{yr}^{-1}$, which is a factor 5-10 higher than that of the fully gravitational model.

We include a realistic gravitational potential into the model, which improves the fit to observations. In this case the SGZ and SNR models give equally good fits to observations.

The influence of magnetic fields and thermal pressure is investigated. If both are included in the model, the turbulent velocity decreases with respect to the case where only the turbulent pressure is taken into account. An increase of the mass accretion rate by a factor 1.5 makes the turbulent velocity dispersion increase again to $10 \mathrm{~km} \mathrm{~s}^{-1}$. In this case the radial profile of the magnetic field is close to observations.

The models nicely reproduce the observed radial dependence of the ratio between the HI and molecular surface densities. We derive a Schmidt law of the form $\dot{\Sigma} \propto \Sigma^{2}$. A possible explanation for the enhanced star formation efficiency observed in a few spiral galaxies is given.

We conclude that for $Q=1$ the models for turbulence (i) driven by $\mathrm{SN}$ energy input and (ii) generated by instabilities involving self-gravitation and maintained by energy input from differential rotation and mass transfer are consistent. The main difference in the disk properties is that $l_{\text {driv }} / H<1$ for case (i) and $l_{\text {driv }} / H=1$ for case (ii).

Both mechanisms are not exclusive and might possibly coexist in galactic disks. A galaxy that slowly forms stars will consume its gas and run into the regime $Q>1$. Then, the timescale for star formation will increase. If locally star formation stops entirely, the turbulent energy will be dissipated within $l_{\text {driv }} / v_{\text {turb }} \sim 10^{7} \mathrm{yr}$, the disk shrinks in $z$ direction and ultimately becomes self-gravitating in $z$. This is the point, where the fully gravitational model of Vollmer \& Beckert (2002) applies. In this case the driving length scale increases and equals the disk height. Thus, the energy dissipation rate, which is proportional to $l_{\text {driv }}^{-2}$ decreases and the small energy supply due to differential rotation is large enough to maintain turbulence.

Such a disk has $Q>1$ and a 5 times lower mass accretion rate than a star forming disk. Once the gas disk is self-gravitating in $z$ it will form stars again and switch to the SGZ/SNR state.

The occurrence of a bar or an external accretion event of a companion can lead to a temporal increase of the mass accretion rate. If $Q$ becomes smaller than 1 during this event, the star formation timescale decreases rapidly. The galaxy will then again reach the regime of $Q \sim 1$. The detailed evolution of such a process can only be investigated by solving the time dependent equation for angular momentum transport.

Acknowledgements. We would like to thank W. J. Duschl and A. Shukurov for fruitful discussions and the anonymous referee for helping us to improve this article significantly.

\section{References}

Allen, C., \& Santillán, A. 1991, RMAA, 22, 255

Avillez, M. A., \& Mac Low, M.-M. 2001, ApJ, 551, L57

Binney, J., \& Tremaine, S. 1987, in Galactic Dynamics (Princeton, University Press)

Blitz, L. 1993, in Protostars and planets III (A93-42937 17-90), 125

Boulares, A., \& Cox, D. P. 1990, ApJ, 365, 544 
Dorfi, E. A. 1993, in Galactic High-Energy Astrophysics. HighAccuracy Timing and Positional Astronomy. Lectures Held at the Astrophysics School IV Organized by the European Astrophysics Doctoral Network (EADN) in Graz, Austria, 19-31 August 1991, ed. J. van Paradijs, \& H. M. Maitzen (Berlin, Heidelberg, New York: Springer-Verlag), 418, 43

Elmegreen, B. G., \& Falgarone, E. 1996, ApJ, 471, 816

Frisch, U. 1995, Turbulence - The Legacy of A. N. Kolmogorov (Cambridge University press)

Gazol-Patino, A., \& Passot, T. 1999, ApJ, 518, 748

Hollenbach, D. J., \& Tielens, A. G. G. M. 1997, ARA\&A, 35, 179

Korpi, M. J., Brandenburg, A., Shukurov, A., Tuominen, I., \& Nordlund, A. 1999, ApJ, 514, L99

Kulkarni, S. R., \& Heiles, C. 1988, in Galactic and Extragalactic Radio Asrtonomy, ed. G. L. Verschurr, \& K. I. Kellermann (Berlin: Springer), 95

Landau, L. D., \& Lifschitz, E. M. 1959, Fluid Mechanics

McKee, C. F. 1995, in The Physics of the Interstellat Medium and the Intergalactic Medium, ed. A. Ferrara, C. Heiles, C. McKee, \& P. Shapiro (San Fransisco: ASP), ASP Conf. Ser., 80, 292
Paczyński, B. 1978, Acta Astron., 28, 91

Prantzos, N., \& Aubert, O. 1995, A\&A, 302, 69

Rosen, A., \& Bregman, J. N. 1995, ApJ, 440, 634

Rownd, B. K., \& Young, J. S. 1999, AJ, 118670

Ruzmaikin, A. A., Shukurov, A. M., \& Sokoloff, D. D. 1988, Magnetic Fields of Galaxies, Astrophysics and space science library, vol. 133 (Kluwer Academic Publishers)

Scalo, J. 1985, in Protostars and Planets II, ed. D. C. Black, \& M. S. Matthews (Tucson: Univ. Arizona), 201

Spitzer, L. 1990, ARA\&A, 28, 71

Strong, A. W., Moskalenko, I. G., \& Reimer, O. 2000, ApJ, 537, 763

Tammann, G. A., Löffler, W., \& Schröder, A. 1994, ApJS, 92, 487

Thornton, K., Gaudlitz, M., Janka, H.-Th., \& Steinmetz, M. 1998, ApJ, 500, 95

Toomre, A. 1964, ApJ, 139, 1217

Vollmer, B., \& Beckert, T. 2002, A\&A, 382, 872

Wada, K., \& Norman, C. A. 2001, ApJ, 547, 172

Wada, K., Meurer, G., \& Norman, C. A. 2002, ApJ, 577, 197

Wong, T., \& Blitz, L. 2000, ApJ, 540, 771

Wong, T., \& Blitz, L. 2002, ApJ, 569, 157 